J. Nonlinear Sci. (2006): pp. OF1-OF40

DOI: $10.1007 / \mathrm{s} 00332-005-0736-\mathrm{Z}$

\title{
Nonlinear
} Science

(C) 2006 Springer Science+Business Media, Inc.

\section{Breakdown of Heteroclinic Orbits for Some Analytic Unfoldings of the Hopf-Zero Singularity}

\author{
I. Baldomá ${ }^{1}$ and T.M. Seara ${ }^{2}$ \\ 1 Departament de Matemàtica Aplicada i Anàlisi, Universitat de Barcelona, Gran Via 587, \\ 08007 Barcelona, Spain \\ e-mail: barraca@mat.ub.es \\ 2 Departament de Matemàtica Aplicada I, Universitat Politècnica de Catalunya, Diagonal 647, \\ 08028 Barcelona, Spain \\ e-mail: tere.m-seara@upc.edu
}

Received September 12, 2005; revised manuscript accepted for publication March 3, 2006

Online publication June 29, 2006

Communicated by G. Haller

Summary. In this paper we study the exponentially small splitting of a heteroclinic connection in a one-parameter family of analytic vector fields in $\mathbb{R}^{3}$. This family arises from the conservative analytic unfoldings of the so-called Hopf zero singularity (central singularity). The family under consideration can be seen as a small perturbation of an integrable vector field having a heteroclinic orbit between two critical points along the $z$ axis. We prove that, generically, when the whole family is considered, this heteroclinic connection is destroyed. Moreover, we give an asymptotic formula of the distance between the stable and unstable manifolds when they meet the plane $z=0$. This distance is exponentially small with respect to the unfolding parameter, and the main term is a suitable version of the Melnikov integral given in terms of the Borel transform of some function depending on the higher-order terms of the family. The results are obtained in a perturbative setting that does not cover the generic unfoldings of the Hopf singularity, which can be obtained as a singular limit of the considered family. To deal with this singular case, other techniques are needed. The reason to study the breakdown of the heteroclinic orbit is that it can lead to the birth of some homoclinic connection to one of the critical points in the unfoldings of the Hopf-zero singularity, producing what is known as a Shilnikov bifurcation.

Key words. Exponentially small splitting, Hopf-zero bifurcation, Melnikov function, Borel transform

\section{Introduction}

One of the most frequently studied problems in the last century was the existence of transversal intersections between stable and unstable manifolds of one or more critical 
points of a dynamical system. This phenomenon is also known as the problem of the splitting of separatrices. Interest in this problem was already noted by Poincaré, who described it as the "fundamental problem of the mechanics" [Poi90]; it is one of the main causes of chaotic behavior. It is well known that the size of the splitting of separatrices gives a measure of the stochastic region of the system.

The most simple setting where this phenomenon occurs is in $T$-periodically perturbed integrable planar systems. In this regular perturbative context, Poincaré, and later Melnikov, constructed a method that allows computation of the splitting of invariant manifolds of hyperbolic critical points which coincide in the unperturbed integrable system. The Poincaré-Melnikov method provides a function whose nondegenerate zeros give rise to transversal homoclinic orbits in the perturbed system; see [Mel63], [GH83]. Several authors have dealt with the problem of generalizing this method to higher dimensional systems. Specifically, for Hamiltonian systems, the (vectorial) Melnikov-Poincaré function turns out to be the gradient of a scalar function which is known as the Melnikov potential. See [Eli94], [DG00] and references therein.

A difficult question arises when this Poincaré-Melnikov function turns out to be exponentially small with respect to the perturbative parameter and hence is not a priori the dominant term. This happens, for instance, in rapidly forced periodic or quasi-periodic perturbations of one-degree-of-freedom Hamiltonian systems, in nearly integrable symplectic mappings that are close to the identity, and in Hamiltonian systems with two or more degrees of freedom which have hyperbolic tori with some fast frequencies, among others.

For some cases of near-integrable time periodic or quasi-periodic Hamiltonian systems, several studies [HMS88], [Sch89], [Ang93], [DS97], [Ge197a], [BF04], [DGJS97] have validated the prediction of the Poincaré-Melnikov function for the splitting and give a rigorous proof of an asymptotic formula for different quantities related to this phenomenon. It is worth mentioning that the Poincaré-Melnikov function does not always give the correct prediction for the splitting; see [Tre97], [Ge197b]. As we have pointed out elsewhere, we can encounter this phenomenon in maps; see [Laz03], [DRR98], [GS01]. The methods developed in these works draw heavily on the Hamiltonian character of the system, especially its symplectic structure.

In this paper we deal with a different setting more related to [Lom99]. We study the splitting of a heteroclinic orbit in a family $X_{\delta}$ (see (1)) of near-integrable analytic vector fields of $\mathbb{R}^{3}$, introduced in Section 1.1.

The family $X_{\delta}$ under consideration becomes, when $\delta=0$, the Hopf-zero singularity (2) also called the central singularity in [GH83]. In fact, in Section 1.2, we show that any generic conservative unfolding of the Hopf-zero singularity can be expressed, after some changes of variables, in a form similar to the family $X_{\delta}$ considered in this paper.

In Section 1.3 we study the relevance of the splitting of the heteroclinic connection for the analytic unfoldings of the Hopf-zero bifurcation. We show that the breakdown of this heteroclinic orbit can lead to the birth of some homoclinic connection in the unfoldings, producing what is known as a Shilnikov bifurcation. In this subsection we also present some results about the existence of Shilnikov bifurcations in the $C^{\infty}$ case. Our final goal is to prove the existence of Shilnikov bifurcations in the analytic unfoldings of the Hopf-zero singularity. We give an asymptotic formula to measure the splitting of the 
heteroclinic connection for analytic families $X_{\delta}$, which turns out to be exponentially small with respect to $\delta$.

Even though the family (1) under consideration can be seen as a perturbation of an integrable conservative vector field, we do not require the perturbation to be conservative at all. For this reason, our proof does not use any geometric structure of the system. The computation of the difference between the stable and unstable manifolds is estimated simply by using the idea in [Sau01], [OSS03], [Bal] that this difference satisfies some linear equation whose solutions can be controlled.

\subsection{Set-Up}

The fields under consideration in this study are of the form:

$$
\begin{aligned}
& \frac{d x}{d \tau}=-\delta x z+y(\alpha+c \delta z)+\delta^{p+1} f(\delta x, \delta y, \delta z, \delta) \\
& \frac{d y}{d \tau}=-\delta y z-x(\alpha+c \delta z)+\delta^{p+1} g(\delta x, \delta y, \delta z, \delta), \\
& \frac{d z}{d \tau}=\delta\left(-1+b\left(x^{2}+y^{2}\right)+z^{2}\right)+\delta^{p+1} h(\delta x, \delta y, \delta z, \delta),
\end{aligned}
$$

where $p \geq-2, \alpha, c, b$ are given constants, $\delta>0$ is a small parameter, and $f, g, h$ are real analytic functions in all their variables whose Taylor series begin at least with terms of degree three.

As we will see in Lemma 1.2, system (1) has a one-dimensional heteroclinic connection, $\{(x, y)=(0,0) ;-1<z<1\}$, between two critical points $(0,0, \pm 1)$ of saddle-focus type when $f=g=h=0$. The goal of this paper is to study the effects of any analytic perturbation $(f, g, h)$ on the invariant stable and unstable manifolds of the critical points of the perturbed system. We will see that generically, if $p>-2$, this heteroclinic connection is destroyed, and moreover, we will compute the distance between the perturbed manifolds when they meet the plane $z=0$. We will prove that such distance, $d^{\mathrm{s}, \mathrm{u}}$, is an exponentially small quantity given by

$$
d^{\mathrm{s}, \mathrm{u}}=C \delta^{p} \mathrm{e}^{-\pi|\alpha| /(2 \delta)}\left(1+O\left(\delta^{p+2}|\log \delta|\right)\right),
$$

for some constant $C$ computed exactly in Theorem 1.4 in terms of the Borel transforms of $f$ and $g$. In particular, if $C \neq 0$ and $\delta>0$ is small enough, we obtain that the heteroclinic connection is destroyed. To state the above result properly, we present the necessary notation in Section 1.5. The rigorous statement is left until to Section 1.6.

Even though we have presented system (1) as a regular perturbation of the case $f=g=h=0$, this system lies in the context of singular perturbation theory. Indeed, when the parameter $\delta=0$, the family of vector fields we are working with becomes

$$
\frac{d x}{d \tau}=\alpha y, \quad \frac{d y}{d \tau}=-\alpha x, \quad \frac{d z}{d \tau}=0 .
$$

Thus, family (1) is a perturbation of system (2). This system is known as the Hopf-zero singularity or the central singularity. 


\subsection{Analytic Unfoldings of the Central Singularity}

Let us consider a vector field in $\mathbb{R}^{3}$ which has the origin as a critical point, and for some positive $\alpha^{*}$, the eigenvalues of the linear part at the origin are $0, \pm \alpha^{*}$. If we assume that the linear part of this vector field is in Jordan normal form, it will be given by

$$
\left(\begin{array}{ccc}
0 & \alpha^{*} & 0 \\
-\alpha^{*} & 0 & 0 \\
0 & 0 & 0
\end{array}\right), \quad \alpha^{*}>0 .
$$

If we consider only the linear context, it is clear that this singularity can be met by a generic family of linear vector fields if it contains at least two parameters. Thus, it has codimension two. But the linear system we are studying has zero divergence; hence it can be also considered in the context of conservative vector fields. In this context it will occur in one-parameter families, and will then have codimension one.

The unfoldings of this singularity have been studied by several authors [Tak73a], [Tak74], [Tak73b], [Guc81], [BV84], [AMF ${ }^{+}$03], [FGRLA02], [DI98], [GH83] looking at the different type of bifurcations that a two (or one) parameter family of vector fields unfolding this singularity can present.

Following [BV84], we present here a description of the singularity we are considering as well as the normal form for the unfoldings of this singularity. Let us then explain the process for obtaining a perturbative setting from the normal form procedure in the more general case.

We consider $X_{\mu, v}$, a family of vector fields on $\mathbb{R}^{3}$ such that $X_{0,0}$ has the origin as a critical point with linear part (3). After some normalization, if we perform the normal form procedure up to order two, we obtain that the vector field $X_{\mu, v}$ in the new coordinates $(\bar{x}, \bar{y}, \bar{z})$ takes the form:

$$
\begin{aligned}
& \frac{d \bar{x}}{d s}=\bar{x}\left(A_{2}(\mu, v)+A_{4}(\mu, v) \bar{z}\right)+\bar{y}\left(A_{1}(\mu, v)+A_{3}(\mu, v) \bar{z}\right)+O_{3}(\bar{x}, \bar{y}, \bar{z}, \mu, v), \\
& \frac{d \bar{y}}{d s}=-\bar{x}\left(A_{1}(\mu, v)+A_{3}(\mu, v) \bar{z}\right)+\bar{y}\left(A_{2}(\mu, v)+A_{4}(\mu, v) \bar{z}\right)+O_{3}(\bar{x}, \bar{y}, \bar{z}, \mu, v), \\
& \frac{d \bar{z}}{d s}=B_{1}(\mu, v)+B_{2}(\mu, v) \bar{z}+B_{3}(\mu, v)\left(\bar{x}^{2}+\bar{y}^{2}\right)+B_{4}(\mu, v) \bar{z}^{2}+O_{3}(\bar{x}, \bar{y}, \bar{z}, \mu, v),
\end{aligned}
$$

where $A_{1}(0,0)=\alpha^{*}, A_{2}(0,0)=B_{1}(0,0)=B_{2}(0,0)=0$. And moreover, after some scaling of the parameters, we can assume that $\partial_{\mu} A_{2}(0,0)=\partial_{\nu} B_{1}(0,0)=0$, $\partial_{\nu} A_{2}(0,0)=1$ and $\partial_{\mu} B_{1}(0,0)=-1$.

The conservative case can be done analogously, considering only one-parameter families with parameter $\mu>0$, and using that, in this case, $B_{2}(\mu)=-2 A_{2}(\mu)$ and $B_{4}(\mu)=-A_{4}(\mu)$. When $\mu>0$, after the scaling $\bar{x}=\delta \tilde{x}, \bar{y}=\delta \tilde{y}, \bar{z}=\delta \tilde{z}, \delta=\sqrt{\mu}$, and calling

$$
a_{j}=A_{j}(0,0), \quad b_{j}=B_{j}(0,0), \quad \text { for } j=3,4,
$$

the system becomes

$$
\frac{d \tilde{x}}{d s}=a_{4} \delta \tilde{x} \tilde{z}+\tilde{y}\left(\alpha^{*}+a_{3} \delta \tilde{z}\right)+\delta^{-1} \tilde{f}(\delta \tilde{x}, \delta \tilde{y}, \delta \tilde{z}, \delta),
$$




$$
\begin{aligned}
& \frac{d \tilde{y}}{d s}=-\tilde{x}\left(\alpha^{*}+a_{3} \delta \tilde{z}\right)+a_{4} \delta \tilde{y} \tilde{z}+\delta^{-1} \tilde{g}(\delta \tilde{x}, \delta \tilde{y}, \delta \tilde{z}, \delta), \\
& \frac{d \tilde{z}}{d s}=\delta\left(-1+b_{3}\left(\tilde{x}^{2}+\tilde{y}^{2}\right)-a_{4} \tilde{z}^{2}\right)+\delta^{-1} \tilde{h}(\delta \tilde{x}, \delta \tilde{y}, \delta \tilde{z}, \delta) .
\end{aligned}
$$

From now on, we will focus our study on the conservative case when $a_{4}<0$. In this case, in order to eliminate the parameter $a_{4}$, we perform the scaling $x=\tilde{x} \sqrt{-a_{4}}$, $y=\tilde{y} \sqrt{-a_{4}}, z=\tilde{z} \sqrt{-a_{4}}$, and, after the change of time $\tau=\sqrt{-a_{4}} s$, system (4) becomes

$$
\begin{aligned}
& \frac{d x}{d \tau}=-\delta x z+(\alpha+c \delta z) y+\delta^{-1} f(\delta x, \delta y, \delta z, \delta), \\
& \frac{d y}{d \tau}=-(\alpha+c \delta z) x-\delta y z+\delta^{-1} g(\delta x, \delta y, \delta z, \delta), \\
& \frac{d z}{d \tau}=-\delta\left(1+b\left(x^{2}+y^{2}\right)+z^{2}\right)+\delta^{-1} h(\delta x, \delta y, \delta z, \delta),
\end{aligned}
$$

where $\alpha=\frac{\alpha^{*}}{\sqrt{-a_{4}}}, c=\frac{a_{3}}{\sqrt{-a_{4}}}$, and $b=\frac{b_{3}}{\sqrt{-a_{4}}}$.

So, if $p=-2$, system (1) under consideration corresponds to the versal unfoldings of the central singularity, also called Hopf-zero bifurcation, after some changes of variables.

\subsection{The Central Singularity and the Shilnikov Bifurcation}

Even though this is not the subject of this paper, let us remember here that a Shilnikov bifurcation occurs when a critical point of saddle-focus type exists and its stable and unstable manifolds intersect, giving rise to the existence of a homoclinic orbit [Šil65], [Šil70], [SSTC01].

In 1984 Broer and Vegter presented, in [BV84], a complete proof of the existence of subordinate Shilnikov bifurcations in generic $\mathcal{C}^{\infty}$ unfoldings of the singularity (3), which have codimension one. The method used in [BV84] to prove the existence of homoclinic orbits is based on the following normal form theorem (see [Bro81a], [Bro81b]):

Theorem 1.1. Let $X=X_{\mu}(x, y, z)$ be a real $\mathcal{C}^{\infty}$ family of vectorfields, where $(x, y, z) \in$ $\mathbb{R}^{3}$ and $\mu \in \mathbb{R}^{k}$. Suppose that for $\mu=0$ the vector field has in $(0,0,0)$ a critical point with linear part (3). Then, there exists a $\mathcal{C}^{\infty} \mu$-dependent change of variables, such that the new vector field becomes $X_{\mu}=\tilde{X}_{\mu}+P$, where $\tilde{X}_{\mu}$, when written in cylindrical coordinates $(r, \varphi, z)$, is

$$
\begin{aligned}
& \frac{d \varphi}{d s}=\tilde{f}\left(r^{2}, z, \mu\right), \\
& \frac{d r}{d s}=r \tilde{g}\left(r^{2}, z, \mu\right), \\
& \frac{d z}{d s}=\tilde{h}\left(r^{2}, z, \mu\right),
\end{aligned}
$$

being $\tilde{f}, \tilde{g}, \tilde{h} C^{\infty}$ functions verifying $\tilde{g}(0,0,0)=\tilde{h}(0,0,0)=\frac{\partial \tilde{h}}{\partial z}(0,0,0)=0$ and $\tilde{f}(0,0,0)=\alpha^{*}$, and the function $P$ is flat at $(x, y, z, \mu)=(0,0,0,0)$. 
In the conservative case, the change can be chosen to be conservative at any order, so $\tilde{X}_{\mu}$ and $P$ have zero divergence. In particular: $\frac{\partial \tilde{f}}{\partial \varphi}+r \frac{\partial \tilde{g}}{\partial r}+\tilde{g}+\frac{\partial \tilde{h}}{\partial z}=0$.

Analyzing the normal form $\tilde{X}_{\mu}$, one can see that it has two hyperbolic critical points of saddle-focus type with a one-dimensional heteroclinic orbit between them. Moreover, for any value of $\mu$ in the conservative case and for a suitable curve in the parameter space in the dissipative case, these points also have a two-dimensional heteroclinic manifold.

By Theorem 1.1 any vector field in the unfolding is given by $X_{\mu}=\tilde{X}_{\mu}+P$. The strategy followed in [BV84] to prove the existence of homoclinic connections is to choose suitable "flat" perturbations $\tilde{P}$ that break the heteroclinic manifolds (both onedimensional and two-dimensional), giving rise to the existence of homoclinic orbits to one of the critical points, for a sequence $\left\{\mu_{n}\right\}_{n}$ going to 0 as $n$ goes to infinity.

More recently, also in the $\mathcal{C}^{\infty}$ context but for reversible systems, a similar result was obtained in [LTW04].

Our final goal is to achieve the same kind of results for analytic unfoldings $X_{\mu}$, where this phenomenon is what is known as a "beyond all orders" or exponentially small phenomenon.

Note that the normal form Theorem 1.1 is not true in the analytic case. For the $\mathcal{C}^{\infty}$ case, there exists not only a formal procedure that casts the system into a formal normal form up to flat terms, but a $\mathcal{C}^{\infty}$ change of variables. This comes from the fact that, even if the formal series obtained are divergent, a classical result in asymptotic series, the Borel-Ritt theorem, gives the existence of $\mathcal{C}^{\infty}$ functions having them as Taylor series.

Of course this reasoning fails in the analytic case, because the function obtained through the Borel-Ritt theorem can not be real analytic if the formal series is divergent. Moreover, for analytic $X_{\mu}$ we can not use "flat" perturbations to break the heteroclinic connections that exist in the normal form, because flat functions are not analytic. However, to prove that these heteroclinic connections are destroyed is a necessary step towards the possible birth of homoclinic orbits.

This paper, in which we deal with the case $p>-2$, is a first contribution to the complete proof of the breakdown of heteroclinic connections which, in our view, is quite delicate and lies in the context of singular perturbation theory.

\subsection{Some Comments about the Singular Case $p=-2$}

As is clear from the above discussion, the generic unfoldings of singularity (3) become system (1) with $p=-2$ after changes of variables and scalings. Nevertheless, it is worthwhile to say that for some (nongeneric) unfoldings we fit in the form (1) for $p>-2$. In fact, system (1) itself is a degenerate unfolding (for instance, among other degenerations, it does not contain second-order terms in its Taylor expansion) of this singularity.

Even if the results of this paper are only rigorously valid for $p>-2$, we hope that it will be possible to adapt some of the methods implemented here to a proof of the exponentially small phenomenon in the limit case $p=-2$.

This limit case, which corresponds to generic unfoldings, is what is known as a singular perturbation case. The reason is the following. The phenomenon we are going 
to study is the splitting of a heteroclinic orbit between two critical points of system (1). As we will see in Section 3, this splitting will be exponentially small with respect to the perturbative parameter ( $\delta$ in our case).

As any expert in this field knows, to give a rigorous proof of an asymptotic formula for this exponentially small splitting, it is necessary to obtain good approximations of the stable and unstable manifolds, not only in the real domain, where they are quite well approximated by the heteroclinic orbit of the unperturbed system, but also in some suitable complex domains.

The perturbation terms $\delta^{-1} f, \delta^{-1} g, \delta^{-1} h$, which in the real domain of the variables are of order $\delta^{2}$, become very big (of order $1 / \delta$ ) when one works in these complex domains where the size of the variables becomes $O(1 / \delta)$. Hence the system is no longer perturbative and, moreover, the manifolds are not close to the unperturbed heterolinic orbit. In this case, matching techniques in the complex plane between different approximations of the manifolds are required to achieve the result. Moreover, contrarily to what happens in the perturbative case $p>-2$ (see Theorem 1.4), the final asymptotic formula will depend on the full jet of the functions $f, g, h$. Examples of rigorous studies of exponentially small phenomena in the singular case can be found in [Gel97a], [Tre97], [OSS03], [RMT97].

To date, only one rigorous proof of the splitting of the one-dimensional heteroclinic connection has been presented, for a special family called the Michelson system [Mic86]:

$$
\begin{aligned}
& \dot{x}=y, \\
& \dot{y}=z, \\
& \dot{z}=c^{2}-\frac{x^{2}}{2}-y .
\end{aligned}
$$

For this system, an unfolding of (3) which has been widely studied (see [Mic86], [JTM92], [KT76], [RMT97]), there is a rigorous proof of an asymptotic formula of the heteroclinic splitting given in [RMT97]. The proof, which falls in the context of singular perturbation theory, draws heavily on the fact that the Michelson system comes from a third-order differential equation. For this reason it is not clear that the methods used can be adapted to generic analytic unfoldings $X_{\mu}$ of the central singularity.

\subsection{Notation and Preliminary Results}

Throughout this paper $|\cdot|$ denotes the maximum norm in $\mathbb{C}^{n}$ :

$$
|z|=\max _{i=1, \ldots, n}\left|z_{i}\right|, \quad z=\left(z_{1}, \ldots, z_{n}\right) \in \mathbb{C}^{n}
$$

and $B\left(r_{0}\right) \subset \mathbb{C}$ denotes the open ball of radius $r_{0}$. We will also use the notation $B^{3}\left(r_{0}\right)=$ $B\left(r_{0}\right) \times B\left(r_{0}\right) \times B\left(r_{0}\right)$.

As usual, we will denote by $\pi^{i}: \mathbb{C}^{3} \rightarrow \mathbb{C}$ the projection over the $i$-component for $i=1,2,3$. Moreover, we will also write $\pi^{i, j}: \mathbb{C}^{3} \rightarrow \mathbb{C}^{2}$ to indicate the projection on the $i, j$ components with $i, j \in\{1,2,3\}$. 
It will be more convenient for our purposes to write system (1) as

$$
\begin{aligned}
& \frac{d x}{d t}=-x z+\left(\frac{\alpha}{\delta}+c z\right) y+\delta^{p} f(\delta x, \delta y, \delta z, \delta), \\
& \frac{d y}{d t}=-\left(\frac{\alpha}{\delta}+c z\right) x-y z+\delta^{p} g(\delta x, \delta y, \delta z, \delta), \\
& \frac{d z}{d t}=-1+b\left(x^{2}+y^{2}\right)+z^{2}+\delta^{p} h(\delta x, \delta y, \delta z, \delta),
\end{aligned}
$$

where $t=\tau \delta, p>-2, f, g$, and $h$ are real analytic functions in $B\left(r_{0}\right)^{3} \times B\left(\delta_{0}\right)$ and, moreover, $f, g, h=O\left(|(x, y, z, \delta)|^{3}\right)$. There is no restriction to assume that $\alpha>0$. We will assume in the sequel that $r_{0}$ is big enough but independent of $\delta$.

Even though system (5) has no meaning for $\delta=0$, when we speak about the unperturbed system we will refer to system (5) with $f=g=h=0$.

The following lemma describes the more relevant geometric facts of the unperturbed system.

Lemma 1.2. The unperturbed system (system (5) with $f=g=h=0$ ) verifies the following, for any value of $\delta>0$ :

1. It possesses only two hyperbolic fixed points $S_{ \pm}^{0}=(0,0, \pm 1)$ which are of saddlefocus type with eigenvalues $\mp 1+\left|\frac{\alpha}{\delta}+c\right| \mathrm{i}, \mp 1-\left|\frac{\alpha}{\delta}+c\right| \mathrm{i}$, and \pm 2 .

2. The one-dimensional unstable manifold of $S_{+}^{0}$ and the one-dimensional stable manifold of $S_{-}^{0}$ coincide along the heteroclinic connection $\{(x, y)=(0,0) ;-1<z<1\}$. This heteroclinic orbit can be parameterized by

$$
\sigma_{0}(t)=(0,0,-\tanh t)
$$

if we require $\sigma_{0}(0)=(0,0,0)$.

3. The polynomial $H(x, y, z)=\frac{x^{2}+y^{2}}{2}\left(z^{2}+\frac{b}{2}\left(x^{2}+y^{2}\right)-1\right)$ is a first integral of the system.

4. If $b>0$, the two-dimensional stable manifold of $S_{+}^{0}$ and the two-dimensional unstable manifold of $S_{-}^{0}$ coincide, giving rise to a two-dimensional heteroclinic surface. Moreover, this heteroclinic surface is given by $z^{2}+\frac{b}{2}\left(x^{2}+y^{2}\right)-1=0$.

Lemma 1.2 describes system (5) as a perturbation of an integrable system. The following result ensures that system (5) has two fixed points of saddle-focus type even when $f, g, h \neq 0$.

Lemma 1.3. If $\delta>0$ is small enough, system (5) has two fixed points $S_{ \pm}(\delta)$ of saddle focus type such that $S_{+}(\delta)$ has a one-dimensional unstable manifold and $S_{-}(\delta)$ has a stable one. We call them $W^{\mathrm{u}, \mathrm{s}}$, respectively.

Moreover, there are no other fixed points of (5) in the closed ball $B\left(\delta^{-1 / 3}\right)$. 
Proof. It is straightforward since we only need to consider the function

$$
P(x, y, z, \delta)=\left(\begin{array}{c}
-x z \delta+(\alpha+\delta c z) y+\delta^{p+1} f(\delta x, \delta y, \delta z, \delta) \\
-y z \delta-(\alpha+\delta c z) x+\delta^{p+1} g(\delta x, \delta y, \delta z, \delta) \\
-1+b\left(x^{2}+y^{2}\right)+z^{2}+\delta^{p} h(\delta x, \delta y, \delta z, \delta)
\end{array}\right) .
$$

It is clear that $\left(x_{0}, y_{0}, z_{0}\right)$ is a fixed point of system (5) if and only if there exists $\delta>0$ such that $P\left(x_{0}, y_{0}, z_{0}, \delta\right)=0$. Hence, since $P(0,0, \pm 1,0)=0$, applying the implicit function theorem we have that there exist neighborhoods of $(0,0, \pm 1), U_{ \pm}, \delta_{0}>0$, and $C^{1}$ functions $S_{ \pm}: B\left(\delta_{0}\right) \rightarrow U_{ \pm}$such that $S_{ \pm}(0)=(0,0, \pm 1)$ and $P(x, y, z, \delta)=0$ if and only if $(x, y, z)=S_{ \pm}(\delta)$. Moreover, one can easily check that $S_{ \pm}(\delta)$ are of saddle-focus type with eigenvalues $\mp 1+\left|\frac{\alpha}{\delta}+c\right| \mathrm{i}+O(\delta), \mp 1-\left|\frac{\alpha}{\delta}+c\right| \mathrm{i}+O(\delta)$, and $\pm 2+O(\delta)$.

To check the second part of the statement, let us assume that there exists a fixed point $(x, y, z) \in B\left(\delta^{-1 / 3}\right)$ of (5). Then, $|f(\delta x, \delta y, \delta z, \delta)|,|g(\delta x, \delta y, \delta z, \delta)| \leq K \delta^{2}$ for some constant $K>0$. Using the triangular inequality,

$$
|\alpha x| \leq K \delta^{p+3}+(1+|c|) \delta^{1 / 3}, \quad|\alpha y| \leq K \delta^{p+3}+(1+|c|) \delta^{1 / 3} .
$$

In addition, taking into account (6), we deduce $\left|-1+z^{2}\right| \leq K \delta^{p+2}+2|b| C \delta^{2 / 3}$ provided that $|h(\delta x, \delta y, \delta z, \delta)| \leq K \delta^{2}$ and $p+3>1$. Henceforth, we have that $|x|,|y|, \mid-1+$ $z^{2} \mid \leq K_{0} \delta^{v}$ with $v=\min \{p+2,1 / 3\}$, and this implies that, taking $\delta$ small enough, $(x, y, z) \in U_{ \pm}$and therefore by the uniqueness of $S_{ \pm},(x, y, z)=S_{ \pm}(\delta)$.

\subsection{Main Result}

By Lemma 1.3, system (5) has two critical points $S_{ \pm}(\delta)$ having one-dimensional stable and unstable manifolds respectively. We are interested in measuring the distance between the stable manifold $W^{\mathrm{s}}$ and the unstable one $W^{\mathrm{u}}$ at the plane $z=0$. We observe that, since system (5) is autonomous, we can fix the origin of time at $t=0$.

Theorem 1.4. Let us consider system (5) with $p>-2$ and $f, g$, and $h$ real analytic functions in $B\left(r_{0}\right)^{3} \times B\left(\delta_{0}\right)$. Moreover, $f, g, h=O\left(|(x, y, z, \delta)|^{3}\right)$. Then, if $\delta>0$ is small enough, we have

1. The one-dimensional stable manifold of $S_{-}(\delta)$ and the one-dimensional unstable manifold of $S_{+}(\delta)$ can be parameterized by $\sigma^{\mathrm{s}}(t, \delta), \sigma^{\mathrm{u}}(t, \delta)$ which are solutions of system (5) such that

$$
\begin{gathered}
\lim _{t \rightarrow \infty} \sigma^{\mathrm{s}}(t, \delta)=S_{-}(\delta), \quad \lim _{t \rightarrow-\infty} \sigma^{\mathrm{u}}(t, \delta)=S_{+}(\delta) \\
\pi^{3} \sigma^{\mathrm{u}}(0, \delta)=\pi^{3} \sigma^{\mathrm{s}}(0, \delta)=0 .
\end{gathered}
$$

2. $\operatorname{Letm}(u)=u^{1+\mathrm{i} c}(f(0,0,-u, 0)+\mathrm{i} g(0,0,-u, 0))=\sum_{n \geq 3} m_{n} u^{n+1+\mathrm{i} c}$ and $\hat{m}(\zeta)=$ $\sum_{n \geq 3} m_{n} \frac{\zeta^{n+\mathrm{i} c}}{\Gamma(n+1+\mathrm{i} c)}$ be its Borel transform.

The difference between the stable and unstable manifolds, $\Delta \sigma(t, \delta)=\sigma^{\mathrm{u}}(t, \delta)-$ $\sigma^{\mathrm{s}}(t, \delta)$, at $t=0$ is given asymptotically by

$$
\Delta \sigma(0, \delta)=\Delta \sigma_{1}(0, \delta)+O\left(\delta^{p+2}|\log \delta|\right) \mathrm{e}^{-|\alpha| \pi /(2 \delta)},
$$




$$
\begin{aligned}
& \text { with } \pi^{3}\left(\Delta \sigma_{1}(0, \delta)\right)=0 \text { and } \\
& \qquad \begin{aligned}
\pi^{1}\left(\Delta \sigma_{1}(0, \delta)\right)+\mathrm{i} \pi^{2}\left(\Delta \sigma_{1}(0, \delta)\right)= & 2 \pi \mathrm{e}^{c \pi / 2} \hat{m}(\mathrm{i} \alpha) \mathrm{e}^{-\mathrm{i} c \log \delta} \delta^{p} \mathrm{e}^{-|\alpha| \pi /(2 \delta)} \\
& +O\left(\delta^{p+1}\right) \mathrm{e}^{-|\alpha| \pi /(2 \delta)} .
\end{aligned}
\end{aligned}
$$

Remark 1.5. Our context could be nonconservative. That is, even if system (5) comes from a conservative context, we do not need this fact in our proof. We do not ask $f, g$, and $h$ to satisfy any additional condition to those stated previously.

Remark 1.6. Even though the distance between the stable and unstable manifold depends on $f, g, h$, and all the parameters of the system, observe that the dominant term for the difference between the stable and unstable manifold depends neither on the function $h$ nor on the parameter $b$.

It is worth mentioning that given $f$ and $g$ such that the Borel transform $\hat{m}$ does not vanish at the point $\mathrm{i} \alpha$, we can state that the heteroclinic connection of system 5 is destroyed.

The proof of this theorem is decomposed in two steps, which are developed in Section 2 and Section 3. Section 2 is devoted to proving the existence of analytic parameterizations of $\sigma^{\mathrm{s}, \mathrm{u}}$ in a suitable complex domain. It is worth mentioning that the parameter we will use is just the time $t$. After that, in Section 3, we will compute the difference between them.

\section{A Parameterization for the Stable and Unstable Manifolds}

The purpose of this section is to provide analytic parameterizations for the stable and unstable manifolds $\sigma^{\mathrm{s}, \mathrm{u}}$, associated with the fixed points $S_{-}(\delta)$ and $S_{+}(\delta)$ respectively, of system (5). These parameterizations are of the form

$$
\sigma^{\mathrm{s}, \mathrm{u}}(t, \delta)=\sigma_{0}(t)+\tilde{\sigma}^{\mathrm{s}, \mathrm{u}}(t, \delta),
$$

and are defined in an appropriate complex domain which we will describe below. The parameter $t$ we will use is just the time.

In order to shorten the notation, we introduce $z_{0}(t)=-\tanh t$ and note that the heteroclinic connection is $\sigma_{0}=\left(0,0, z_{0}\right)$.

In Section 2.1 we find the differential equation that $\tilde{\sigma}^{\text {s,u }}$ have to satisfy and we perform a complex change of variables in order to put the linear part of this differential equation in diagonal form. We will use this new system throughout the remaining part of the paper. After that, in Section 2.2, we introduce some functional spaces with which we will work in this section. Finally, in Section 2.3, we prove the existence and some useful properties of the parameterizations of the stable and unstable manifolds $\sigma^{\mathrm{s}, \mathrm{u}}$ by using a suitable version of the fixed point theorem.

\subsection{A Preliminary Change of Coordinates}

In this subsection, we will write system (5) in a more appropriate way. 
Since we are looking for parameterizations of the stable and unstable manifolds of the form (7), as usual, we perform the time-dependent change of coordinates given by $(u, v, w, t)=C_{0}(x, y, z, t)=\left(x, y, z-z_{0}(t), t\right)$. For simplicity, we also perform the change of variables given by $(\xi, \bar{\xi}, \eta)=C_{1}(u, v, w)=(u+\mathrm{i} v, u-\mathrm{i} v, w)$ in order to put the linear part of the new system in diagonal form. After these changes, system (5) becomes

$$
\begin{aligned}
& \dot{\xi}=-\left(\frac{\alpha}{\delta}+c z_{0}(t)\right) \mathrm{i} \xi-\xi z_{0}(t)-(1+\mathrm{i} c) \eta \xi+\delta^{p} F_{1}\left(\delta \xi, \delta \bar{\xi}, \delta\left(z_{0}(t)+\eta\right), \delta\right), \\
& \dot{\bar{\xi}}=\left(\frac{\alpha}{\delta}+c z_{0}(t)\right) \mathrm{i} \bar{\xi}-\bar{\xi} z_{0}(t)-(1-\mathrm{i} c) \eta \bar{\xi}+\delta^{p} F_{2}\left(\delta \xi, \delta \bar{\xi}, \delta\left(z_{0}(t)+\eta\right), \delta\right), \\
& \dot{\eta}=2 z_{0}(t) \eta+b \xi \bar{\xi}+\eta^{2}+\delta^{p} H\left(\delta \xi, \delta \bar{\xi}, \delta\left(z_{0}(t)+\eta\right), \delta\right),
\end{aligned}
$$

where $\cdot=\frac{d}{d t}$ and $F=\left(F_{1}, F_{2}\right)$, with

$$
\begin{aligned}
F_{1}(\delta \xi, \delta \bar{\xi}, \delta \eta, \delta) & =(f+\mathrm{i} g)\left(\delta C_{1}^{-1}(\xi, \bar{\xi}, \eta), \delta\right) \\
F_{2}(\delta \xi, \delta \bar{\xi}, \delta \eta, \delta) & =(f-\mathrm{i} g)\left(\delta C_{1}^{-1}(\xi, \bar{\xi}, \eta), \delta\right) \\
H(\delta \xi, \delta \xi, \delta \eta, \delta) & =h\left(\delta C_{1}^{-1}(\xi, \bar{\xi}, \eta), \delta\right)
\end{aligned}
$$

We write $\zeta=(\xi, \bar{\xi}, \eta)$, and we define

$$
\begin{aligned}
\mathcal{R} & =(\mathcal{M}, \mathcal{N}), \\
\mathcal{M}(\zeta) & =\left(\begin{array}{c}
\xi \eta(-1-\mathrm{i} c) \\
\bar{\xi} \eta(-1+\mathrm{i} c)
\end{array}\right)+\delta^{p} F\left(\delta \xi, \delta \bar{\xi}, \delta\left(z_{0}(t)+\eta\right), \delta\right), \\
\mathcal{N}(\zeta) & =b \xi \bar{\xi}+\eta^{2}+\delta^{p} H\left(\delta \xi, \delta \bar{\xi}, \delta\left(z_{0}(t)+\eta\right), \delta\right),
\end{aligned}
$$

and the matrix

$$
A(t)=\left(\begin{array}{ccc}
-\left(\frac{\alpha}{\delta}+c z_{0}(t)\right) \mathrm{i}-z_{0}(t) & 0 & 0 \\
0 & \left(\frac{\alpha}{\delta}+c z_{0}(t)\right) \mathrm{i}-z_{0}(t) & 0 \\
0 & 0 & 2 z_{0}(t)
\end{array}\right)
$$

It is clear that, with this notation, system (8) can simply be written as

$$
\dot{\zeta}=A(t) \zeta+\mathcal{R}(\zeta)
$$

Lemma 2.1. The fundamental matrix, $\Phi$, of system $\dot{\zeta}=A(t) \zeta$ satisfying that $\Phi(0)=$ Id is given by

$$
\Phi(t)=\left(\begin{array}{ccc}
\cosh t \mathrm{e}^{-\mathrm{i} \alpha t / \delta} \mathrm{e}^{\mathrm{i} c \log (\cosh t)} & 0 & 0 \\
0 & \cosh t \mathrm{e}^{\mathrm{i} \alpha t / \delta} \mathrm{e}^{-\mathrm{i} c \log (\cosh t)} & 0 \\
0 & 0 & \cosh ^{-2} t
\end{array}\right) .
$$




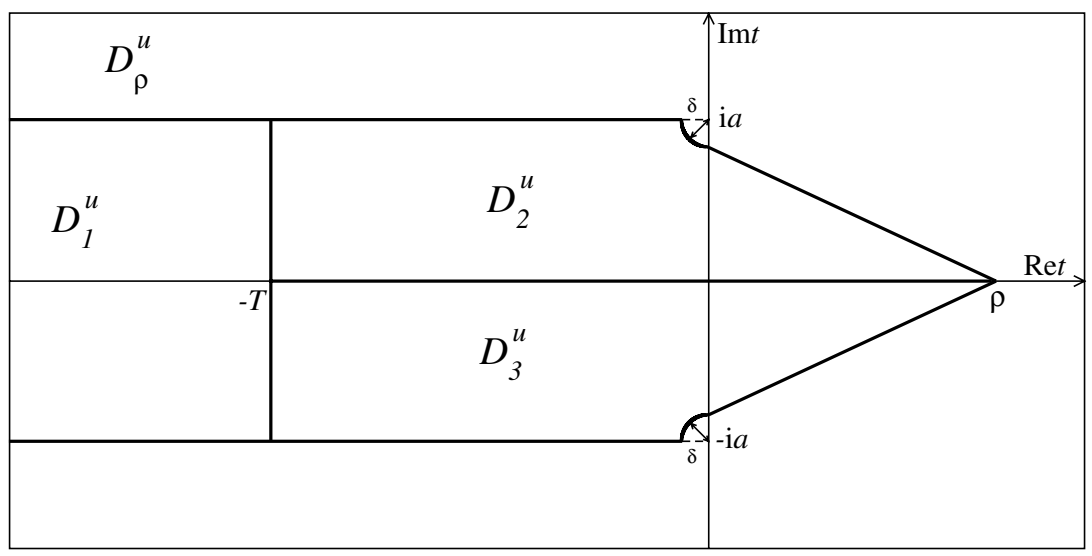

Fig. 1. Defined sets.

\subsection{Domains and Functional Spaces}

This subsection is mainly devoted to introducing the Banach spaces we will use throughout this section.

Let us recall that $\sigma_{0}(t)$, the heteroclinic orbit of the unperturbed system, is given by $\sigma_{0}(t)=\left(0,0, z_{0}(t)\right)$. The function $z_{0}(t)=-\tanh t$ is a real analytic function, which has poles in $t= \pm \frac{\pi}{2} \mathrm{i}+2 k \pi \mathrm{i}$. In order to achieve the desired results, we will need to work in a suitable complex domain that reaches a small neighborhood of the first singularities $\pm \mathrm{i} \pi / 2$. For any $\rho>0$, we define the complex domain:

$$
\begin{aligned}
D_{\rho}^{\mathrm{u}}= & \{t \in \mathbb{C}:|\operatorname{Im} t| \leq a, \operatorname{Re} t \leq-\delta\} \\
& \cup\left\{t \in \mathbb{C}:|\operatorname{Im} t| \leq(a-\delta)\left(1-\frac{\operatorname{Re} t}{\rho}\right), \operatorname{Re} t \geq 0\right\} \\
& \cup\left\{t \in \mathbb{C}:-\delta \leq \operatorname{Re} t \leq 0|\operatorname{Im} t| \leq a-\sqrt{\delta^{2}-(\operatorname{Re} t)^{2}}\right\},
\end{aligned}
$$

where $a=\frac{\pi}{2}$. We take $T>2 \log 2$ and we decompose $D_{\rho}^{\mathrm{u}}=D_{1}^{\mathrm{u}} \cup D_{2}^{\mathrm{u}} \cup D_{3}^{\mathrm{u}}$ where $D_{i}^{\mathrm{u}}$ for $i=1,2,3$, are the sets defined by Figure 1 . That is,

$$
\begin{aligned}
& D_{1}^{\mathrm{u}}=\left\{t \in D_{\rho}^{\mathrm{u}}: \operatorname{Re} t \leq-T\right\}, \\
& D_{2}^{\mathrm{u}}=\left\{t \in D_{\rho}^{\mathrm{u}}: \operatorname{Re} t \geq-T \text { and } \operatorname{Im} t \geq 0\right\}, \\
& D_{3}^{\mathrm{u}}=\left\{t \in D_{\rho}^{\mathrm{u}}: \operatorname{Re} t \geq-T \text { and } \operatorname{Im} t \leq 0\right\} .
\end{aligned}
$$

Analogously, we denote

$$
D_{\rho}^{\mathrm{s}}=\left\{t \in \mathbb{C}:-t \in D_{\rho}^{\mathrm{u}}\right\}, \quad D_{i}^{\mathrm{s}}=\left\{t \in \mathbb{C}:-t \in D_{i}^{\mathrm{u}}\right\}, \quad \text { for } i=1,2,3 .
$$

All the functions we will discuss will depend on $\delta$ as a parameter. If there is no danger of confusion, we will skip this dependence in our notation, and we will take $|\delta|<\delta_{0}$. 
For any $v \geq 0$, we introduce $\mathcal{X}_{v}^{\mathrm{u}}$, the space of analytic functions such that $f$ belongs to $\mathcal{X}_{v}^{\mathrm{u}}$ if and only if

1. $f: \operatorname{int}\left(D_{\rho}^{\mathrm{u}}\right) \rightarrow \mathbb{C}$ is continuous and analytic in $\circ \operatorname{int}\left(D_{\rho}^{\mathrm{u}}\right)$.

2. $f$ satisfies that

$$
\sup _{t \in D_{1}^{\mathrm{u}}}|f(t)|+\sup _{t \in D_{2}^{\mathrm{u}}}\left|(t-\mathrm{i} a)^{\nu} f(t)\right|+\sup _{t \in D_{3}^{\mathrm{u}}}\left|(t+\mathrm{i} a)^{\nu} f(t)\right|<+\infty .
$$

We endow $\mathcal{X}_{v}^{\mathrm{u}}$ with the norm:

$$
\|f\|_{v}=\sup _{t \in D_{1}^{\mathrm{u}}}|f(t)|+\sup _{t \in D_{2}^{\mathrm{u}}}\left|(t-\mathrm{i} a)^{v} f(t)\right|+\sup _{t \in D_{3}^{\mathrm{u}}}\left|(t+\mathrm{i} a)^{v} f(t)\right| .
$$

With this norm, $\mathcal{X}_{v}^{\mathrm{u}}$ becomes a Banach space. We also introduce

$$
\mathcal{X}_{v}^{\mathrm{s}}=\left\{f: D_{\rho}^{\mathrm{s}} \rightarrow \mathbb{C} \text { such that } g_{f} \text { defined by } g_{f}(t):=f(-t) \text { belongs to } \mathcal{X}_{v}^{\mathrm{u}}\right\}
$$

Trivially, $\mathcal{X}_{v}^{\mathrm{s}}$ is a Banach space with the norm $\|f\|_{\nu}^{\mathrm{s}}=\left\|g_{f}\right\|_{\nu}$.

From now on, if there is no danger of confusion, we will also denote $\|\cdot\|_{v}^{\mathrm{s}}$ simply by $\|\cdot\|_{\nu}$.

Remark 2.2. If $v_{1} \leq v_{2}$, then $\mathcal{X}_{v_{1}}^{\mathrm{s}, \mathrm{u}} \subset \mathcal{X}_{v_{2}}^{\mathrm{s}, \mathrm{u}}$, and moreover, there exists a constant $K=K(T, a)$ such that for all $f \in \mathcal{X}_{v_{1}}^{\text {s,u }}$,

$$
\|f\|_{\nu_{2}} \leq K\|f\|_{\nu_{1}} \text {. }
$$

Proof. We fix $f \in \mathcal{X}_{v_{1}}^{\mathrm{u}}$ (the case $f \in \mathcal{X}_{v_{1}}^{\mathrm{s}}$ is analogous). Let $t \in D_{2}^{\mathrm{u}}$. Then

$$
|t-\mathrm{i} a|^{\nu_{2}}|f(t)| \leq|t-\mathrm{i} a|^{\nu_{2}-v_{1}}\|f\|_{\nu_{1}} \leq\left((\max \{\rho, T\})^{2}+a^{2}\right)^{\left(\nu_{2}-v_{1}\right) / 2}\|f\|_{\nu_{1}} .
$$

We denote $C=\left((\max \{\rho, T\})^{2}+a^{2}\right)^{\left(\nu_{2}-v_{1}\right) / 2}$. In the same way one can check that, if $t \in D_{3}^{\mathrm{u}},|t+\mathrm{i} a|^{\nu_{2}}|f(t)| \leq C\|f\|_{\nu_{1}}$, and hence $f \in \mathcal{X}_{\nu_{2}}^{\mathrm{u}}$ and

$$
\|f\|_{\nu_{2}} \leq(1+2 C)\|f\|_{\nu_{1}} .
$$

For technical reasons, we endow the product space $\mathcal{X}_{3}^{\mathrm{s}, \mathrm{u}} \times \mathcal{X}_{3}^{\mathrm{s}, \mathrm{u}} \times \mathcal{X}_{2}^{\mathrm{s}, \mathrm{u}}$ with the norm

$$
\begin{aligned}
\|f\| & =\left\|f_{1}\right\|_{3}+\left\|f_{2}\right\|_{3}+\delta|\log \delta|^{-1}\left\|f_{3}\right\|_{2}, \\
f & =\left(f_{1}, f_{2}, f_{3}\right) \in \mathcal{X}_{3}^{\mathrm{s}, \mathrm{u}} \times \mathcal{X}_{3}^{\mathrm{s}, \mathrm{u}} \times \mathcal{X}_{2}^{\mathrm{s}, \mathrm{u}} .
\end{aligned}
$$

We will also use the norm

$$
\|f\|_{\times, v}=\left\|f_{1}\right\|_{\nu}+\left\|f_{2}\right\|_{\nu}, \quad f=\left(f_{1}, f_{2}\right) \in \mathcal{X}_{\nu}^{\mathrm{s}, \mathrm{u}} \times \mathcal{X}_{\nu}^{\mathrm{s}, \mathrm{u}} .
$$

\subsection{Analytic Parameterization of the Invariant Manifolds}

In this subsection we prove that equation (13),

$$
\dot{\zeta}=A(t) \zeta+\mathcal{R}(\zeta)
$$


has solutions, $\varphi^{\mathrm{s}, \mathrm{u}}$, defined in $D_{\rho}^{\mathrm{s}, \mathrm{u}}$ satisfying that

$$
\pi^{3}\left(\varphi^{\mathrm{s}, \mathrm{u}}(0)\right)=0 \quad \text { and } \quad \sup _{t \in D_{\rho}^{\mathrm{s}, \mathrm{u}}}\left|\varphi^{\mathrm{s}, \mathrm{u}}(t)\right| \leq M
$$

If $\varphi^{\mathrm{s}, \mathrm{u}}$ satisfy these conditions, the parameterizations $\sigma^{\mathrm{s}, \mathrm{u}}$ of the stable and unstable manifolds we are looking for are given by $\sigma^{\mathrm{s}, \mathrm{u}}=\sigma_{0}+C_{1}^{-1}\left(\varphi^{\mathrm{s}, \mathrm{u}}\right)$. Indeed, Lemma 1.3 says that system (5) has stable and unstable manifolds associated with the fixed points $S_{+}(\delta)$ and $S_{-}(\delta)$. Moreover, according to hyperbolic theory, the only bounded solutions are in $B\left(\delta^{-1 / 3}\right)$.

In order to prove the existence and properties of the solutions $\varphi^{\mathrm{s}, \mathrm{u}}$ of system (13), our strategy will be to use a suitable version of the fixed point theorem in the Banach spaces $\mathcal{X}_{3}^{\mathrm{s}, \mathrm{u}} \times \mathcal{X}_{3}^{\mathrm{s}, \mathrm{u}} \times \mathcal{X}_{2}^{\mathrm{s}, \mathrm{u}}$. To this end, our first step will be to find a fixed point equation for $\varphi^{\mathrm{u}, \mathrm{s}}$.

Let us consider the following linear operators, acting on functions $\phi: D_{\rho}^{\mathrm{s}, \mathrm{u}} \rightarrow \mathbb{C}$ :

$$
\begin{aligned}
\mathcal{L}_{\alpha, c}^{\mathrm{s}, \mathrm{u}}(\phi)(t) & =\cosh t \int_{ \pm \infty}^{0} \frac{1}{\cosh (t+r)} \mathrm{e}^{\mathrm{i} \alpha r / \delta} \mathrm{e}^{\mathrm{i} c(\log \cosh t-\log \cosh (t+r))} \phi(t+r) d r \\
\mathcal{T}(\phi)(t) & =\frac{1}{\cosh ^{2} t} \int_{0}^{t} \cosh ^{2} r \phi(r) d r
\end{aligned}
$$

where in $(20)+$ stands for s and - stands for $\mathrm{u}$. We also define the linear operator $\mathcal{S}^{\mathrm{s}, \mathrm{u}}$ given by

$$
\mathcal{S}^{\mathrm{s}, \mathrm{u}}(\psi)=\left(\mathcal{L}_{\alpha, c}^{\mathrm{s}, \mathrm{u}}\left(\pi^{1} \psi\right), \mathcal{L}_{-\alpha,-c}^{\mathrm{s}, \mathrm{u}}\left(\pi^{2} \psi\right)\right), \quad \psi: D_{\rho}^{\mathrm{s}, \mathrm{u}} \rightarrow \mathbb{C}^{2}
$$

and finally the operator $\mathcal{L}^{\mathrm{s}, \mathrm{u}}$ by

$$
\mathcal{L}^{\mathrm{s}, \mathrm{u}}(\chi)=\left(\mathcal{S}^{\mathrm{s}, \mathrm{u}} \circ \pi^{1,2}(\chi), \mathcal{T} \circ \pi^{3}(\chi)\right), \quad \chi: D_{\rho}^{\mathrm{s}, \mathrm{u}} \rightarrow \mathbb{C}^{3}
$$

Lemma 2.3. With the above notation, if a bounded and continuous function $\varphi^{\mathrm{s}, \mathrm{u}}: D_{\rho}^{\mathrm{s}, \mathrm{u}} \rightarrow$ $\mathbb{C}^{3}$ satisfies the fixed point equation

$$
\varphi^{\mathrm{s}, \mathrm{u}}=\mathcal{L}^{\mathrm{s}, \mathrm{u}} \circ \mathcal{R}\left(\varphi^{\mathrm{s}, \mathrm{u}}\right),
$$

then it is a solution of (13) satisfying (19).

Proof. The proof is straightforward; we only have to differentiate with respect to $t$ equation (24).

Remark 2.4. The choice of the linear operators $\mathcal{L}^{\mathrm{s}, \mathrm{u}}$ is, in some sense, natural. Indeed, by Lemma 2.1, any solution $\varphi$ of equation (13) must satisfy the integral equation

$$
\varphi(t)=\Phi(t)\left[\varphi(0)+\int_{0}^{t} \Phi^{-1}(s) \mathcal{R}(\varphi(s)) d s\right]
$$


(The fundamental matrix $\Phi$ was defined in Lemma 2.1.) Since we are looking for solutions satisfying the properties (19), $\varphi^{\mathrm{s}, \mathrm{u}}$ must satisfy the integral equation

$$
\varphi^{\mathrm{s}, \mathrm{u}}(t)=\left(\begin{array}{l}
\cosh t \int_{ \pm \infty}^{t} \frac{1}{\cosh r} \mathrm{e}^{-\mathrm{i} \alpha(t-r) / \delta} \mathrm{e}^{\mathrm{i} c\left(\log \left(\frac{\cosh t}{\cosh r}\right)\right)} \pi^{1}\left(\mathcal{M}\left(\varphi^{\mathrm{s}, \mathrm{u}}(r)\right)\right) d r \\
\cosh t \int_{ \pm \infty}^{t} \frac{1}{\cosh r} \mathrm{e}^{\mathrm{i} \alpha(t-r) / \delta} \mathrm{e}^{-\mathrm{i} c\left(\log \left(\frac{\cosh t}{\cosh r}\right)\right)} \pi^{2}\left(\mathcal{M}\left(\varphi^{\mathrm{s}, \mathrm{u}}(r)\right)\right) d r \\
\frac{1}{\cosh ^{2} t} \int_{0}^{t} \cosh ^{2} r \mathcal{N}\left(\varphi^{\mathrm{s}, \mathrm{u}}(r)\right) d r
\end{array}\right)
$$

taking the + sign for $\mathrm{s}$ and - for $\mathrm{u}$. We observe that the third component of (25) is identical to the third component of equation (24). Finally, one can easily check that, changing the integration path to $\gamma(t)=t+s, s \in( \pm \infty, 0]$ and using Cauchy's theorem, the first and second components of (25) are actually $\mathcal{S}^{\mathrm{s}, \mathrm{u}} \circ \mathcal{M}\left(\varphi^{\mathrm{s}, \mathrm{u}}\right)$.

The remaining part of this subsection is devoted to proving the following proposition:

Proposition 2.5. If $p>-2$ and $\delta$ is small enough, system (13) has two solutions $\varphi^{\mathrm{s}, \mathrm{u}}$ satisfying that $\varphi^{\mathrm{s}, \mathrm{u}}=\varphi_{1}^{\mathrm{s}, \mathrm{u}}+\varphi_{2}^{\mathrm{s}, \mathrm{u}}$, with $\varphi_{1}^{\mathrm{s}, \mathrm{u}}$ and $\varphi_{2}^{\mathrm{s}, \mathrm{u}}$ having the following properties:

1. $\varphi_{1}^{\mathrm{s}, \mathrm{u}}=\mathcal{L}^{\mathrm{s}, \mathrm{u}} \circ \mathcal{R}(0) \in \mathcal{X}_{3}^{\mathrm{s}, \mathrm{u}} \times \mathcal{X}_{3}^{\mathrm{s}, \mathrm{u}} \times \mathcal{X}_{2}^{\mathrm{s}, \mathrm{u}}$ and $\left\|\varphi_{1}^{\mathrm{s}, \mathrm{u}}\right\| \leq K \delta^{p+4}$,

2. $\varphi_{2}^{\mathrm{s}, \mathrm{u}} \in \mathcal{X}_{3}^{\mathrm{s}, \mathrm{u}} \times \mathcal{X}_{3}^{\mathrm{s}, \mathrm{u}} \times \mathcal{X}_{2}^{\mathrm{s}, \mathrm{u}}$ and $\left\|\varphi_{2}^{\mathrm{s}, \mathrm{u}}\right\| \leq K \delta^{p+2}|\log \delta|\left\|\varphi_{1}^{\mathrm{s}, \mathrm{u}}\right\|$,

for some constant $K$ independent of $\delta$.

Remark 2.6. We note that, by Lemma 2.3, we are allowed to use the fixed point equation (24) to prove Proposition 2.5.

The proof of Proposition 2.5 is broken down into three steps, which are developed in the subsections below. In Section 2.3.1, we prove that the linear operators $\mathcal{S}^{\mathrm{s}, \mathrm{u}}$ and $\mathcal{T}$ are continuous in suitable Banach spaces. After that, in Section 2.3.2, we will give the properties of $\varphi_{1}^{\mathrm{s}, \mathrm{u}}$ enunciated in Proposition 2.5. Finally, we complete the proof by using an appropriate version of the fixed point theorem. This last step is done in Section 2.3.3.

From now on we only deal with the unstable manifold $\varphi^{\mathrm{u}}$. For this reason we will skip the -u- sign of our notation, writing, for instance, $\varphi, \mathcal{S}, D_{\rho}$, and $D_{i}$ instead of $\varphi^{\mathrm{u}}$, $\mathcal{S}^{\mathrm{u}}, D_{\rho}^{\mathrm{u}}$, and $D_{i}^{\mathrm{u}}$, respectively.

In the remaining part of this section, we will make particular use of the geometry of the domain $D_{\rho}$ defined in (14) and its decomposition $D_{\rho}=D_{1} \cup D_{2} \cup D_{3}$ given in (15). See also Figure 1.

2.3.1. The Linear Operators $\mathcal{S}$ and $\mathcal{T}$. Here we will study the linear operators $\mathcal{S}$ and $\mathcal{T}$. First of all we enunciate a technical lemma; see ([DS97]). 
Lemma 2.7. The following bounds hold:

1. Let $v \geq 1$. There exists a constant $C=C(\rho, T)$ such that, if $s \in D_{2}$ and $s_{0} \in \mathbb{R}$,

$$
\left|\int_{0}^{s_{0}}\right| s+r-\left.\mathrm{i} a\right|^{-v} d r \mid \leq C \begin{cases}\sup _{r \in\left[0, s_{0}\right]}|s+r-\mathrm{i} a|^{-v+1} & \text { if } v>1 \\ \sup _{r \in\left[0, s_{0}\right]} \log |s+r-\mathrm{i} a| & \text { if } v=1\end{cases}
$$

moreover,

$$
\left|\int_{0}^{s}\right| r-\left.\mathrm{i} a\right|^{-1} d r|\leq C| \log \delta \mid .
$$

2. There exist constants $K_{1}, K_{2}>0$ depending on $T, \rho$ such that

$$
\begin{aligned}
& K_{1}|t-\mathrm{i} a| \leq|\cosh t| \leq K_{2}|t-\mathrm{i} a|, \quad t \in D_{2}, \\
& K_{1}|t+\mathrm{i} a| \leq|\cosh t| \leq K_{2}|t+\mathrm{i} a|, \quad t \in D_{3} .
\end{aligned}
$$

3. Let $T>2 \log 2$. If $t \in D_{1}$ and $r<0$, then

$$
|\cosh (t+r)| \geq|\sinh (\operatorname{Re} t+r)| \geq \frac{\mathrm{e}^{|\operatorname{Re} t+r|}}{4} .
$$

Moreover, we also have that for all $t \in \mathbb{C},|\cosh t| \leq \cosh (\operatorname{Re} t) \leq \mathrm{e}^{|\operatorname{Re} t|}$.

The next lemma studies the linear operator $\mathcal{S}$.

Lemma 2.8. For any $v>1$ and $T$ big enough, the operator $\mathcal{S}: \mathcal{X}_{v} \times \mathcal{X}_{v} \rightarrow \mathcal{X}_{v-1} \times \mathcal{X}_{v-1}$ given in (22) is well defined and there exists a constant $K$ independent of $\delta$ such that

$$
\|\mathcal{S}(\psi)\|_{\times, v-1} \leq K\|\psi\|_{\times, \nu}, \quad \text { for any } \psi \in \mathcal{X}_{\nu} \times \mathcal{X}_{\nu}
$$

Proof. First we observe that if $t \in D_{\rho}$, then $|\operatorname{Im}(\log (\cosh t))| \leq \pi / 2$. Hence, since $t+r \in D_{\rho}$ if $t \in D_{\rho}$ and $r \leq 0$, we have that

$$
\left|\mathrm{e}^{\mathrm{i} c(\log \cosh t-\log \cosh (t+r))}\right| \leq \mathrm{e}^{|c| \pi} .
$$

Let $\psi \in \mathcal{X}_{v} \times \mathcal{X}_{\nu}$. By definition of $\mathcal{S}$, it is enough to check that $\left\|\mathcal{L}_{\alpha, c}\left(\pi^{1} \psi\right)\right\|_{\nu-1} \leq$ $K\left\|\pi^{1} \psi\right\|_{\nu}$ and $\left\|\mathcal{L}_{-\alpha,-c}\left(\pi^{2} \psi\right)\right\|_{\nu-1} \leq K\left\|\pi^{2} \psi\right\|_{\nu}$ for some constant $K$.

We deal only with $\pi^{1} \psi$; the other case is analogous. We denote $\phi=\pi^{1} \psi \in \mathcal{X}_{v}$. Using inequality (26) and definition (20) of $\mathcal{L}_{\alpha, c}(\phi)$, we obtain that, for any $t \in D_{\rho}$,

$$
\left|\mathcal{L}_{\alpha, c}(\phi)(t)\right| \leq \mathrm{e}^{|c| \pi}|\cosh t| \int_{-\infty}^{0} \frac{|\phi(t+r)|}{|\cosh (t+r)|} d r:=\mathrm{e}^{|c| \pi} I(t) .
$$

Now we will bound $I(t)$. For that purpose we will distinguish three cases according to the $D_{i}$ where $t$ belongs to (see Figure 1).

If $t \in D_{1}$, then $t+r \in D_{1}$ for all $r<0$, and hence $|\phi(t+r)| \leq\|\phi\|_{\nu}$. Using (3) of Lemma 2.7 to bound $I(t)$ in (27), we get that

$$
I(t) \leq 4\|\phi\|_{\nu} \mathrm{e}^{|\operatorname{Re} t|} \int_{-\infty}^{0} \mathrm{e}^{-|\operatorname{Re} t+r|} d r=4\|\phi\|_{\nu} .
$$


Now we deal with the case when $t \in D_{2}$. Then $t+r \in D_{2}$ if $r \in[-T-\operatorname{Re} t, 0]$, and $t+r \in D_{1}$ if $r<-T-\operatorname{Re} t$. Hence, in order to bound $I(t)$, we have to decompose it in two parts, that is,

$$
\begin{aligned}
I(t) & =I_{1}(t)+I_{2}(t) \\
& :=|\cosh t| \int_{-\infty}^{-T-\operatorname{Re} t} \frac{|\phi(t+r)|}{|\cosh (t+r)|} d r+|\cosh t| \int_{-T-\operatorname{Re} t}^{0} \frac{|\phi(t+r)|}{|\cosh (t+r)|} d r .
\end{aligned}
$$

First we deal with $I_{1}(t)$. It is clear that

$$
\begin{aligned}
I_{1}(t) & =\frac{|\cosh t|}{|\cosh (-T+\mathrm{i} \operatorname{Im} t)|}|\cosh (-T+\mathrm{i} \operatorname{Im} t)| \int_{-\infty}^{0} \frac{|\phi(-T+\mathrm{i} \operatorname{Im} t+r)|}{|\cosh (-T+\mathrm{i} \operatorname{Im} t+r)|} d r \\
& =\frac{|\cosh t|}{|\cosh (-T+\mathrm{i} \operatorname{Im} t)|} I(-T+\mathrm{i} \operatorname{Im} t) .
\end{aligned}
$$

Hence, since $-T+\mathrm{i} \operatorname{Im} t \in D_{1}$, we can use (28) to bound $I(-T+\mathrm{i} \operatorname{Im} t)$. Moreover, again using (3) from Lemma 2.7, we have that

$$
I_{1}(t) \leq 16 \mathrm{e}^{|\operatorname{Re} t|-T}\|\phi\|_{\nu} \leq 16 \mathrm{e}^{\max \{0, \rho-T\}}\|\phi\|_{\nu},
$$

provided $|\operatorname{Re} t| \leq \max \{T, \rho\}$.

Next we deal with $I_{2}(t)$. As we pointed out before, since $t \in D_{2}$ and $r \in[-T-$ $\operatorname{Re} t, 0], t+r \in D_{2}$, and hence $|\phi(t+r)| \leq\|\phi\|_{v}|t+r-\mathrm{i} a|^{-v}$. Thus, using (1) and (2) of Lemma 2.7 to bound $I_{2}(t)$, we have that

$$
\begin{aligned}
I_{2}(t) & \leq K_{2} K_{1}^{-1}\|\phi\|_{\nu}|t-\mathrm{i} a| \int_{-T-\operatorname{Re} t}^{0}|t+r-\mathrm{i} a|^{-v-1} d r \\
& \leq C K_{2} K_{1}^{-1}\|\phi\|_{\nu}|t-\mathrm{i} a| \sup _{r \in[-T-\operatorname{Re} t, 0]}|t+r-\mathrm{i} a|^{-v} .
\end{aligned}
$$

Let $C_{\rho}=\left(1+\rho^{2} /(a-\delta)^{2}\right)^{1 / 2}$. First we note that if $t \in D_{2}$ and $\operatorname{Re} t>0$, then $|t-\mathrm{i} a| \leq C_{\rho}|\operatorname{Im} t-a|$. Next we observe that, if $t \in D_{2}$ and $r \in[-T-\operatorname{Re} t, 0]$,

$$
\begin{aligned}
& |t+r-\mathrm{i} a| \geq|t-\mathrm{i} a|, \quad \text { if } \operatorname{Re} t \leq 0 \\
& |t+r-\mathrm{i} a| \geq|\operatorname{Im} t-a| \geq C_{\rho}^{-1}|t-\mathrm{i} a|, \quad \text { if } \operatorname{Re} t>0 .
\end{aligned}
$$

Therefore, since $C_{\rho}^{-1} \leq 1$, we have that $|t+r-\mathrm{i} a| \geq C_{\rho}^{-1}|t-\mathrm{i} a|$ for all $t \in D_{2}$ and $r \in[-T-\operatorname{Re} t, 0]$. Thus we obtain

$$
I_{2}(t) \leq C K_{2} K_{1}^{-1} C_{\rho}^{v}\|\phi\|_{v}|t-\mathrm{i} a|^{-v+1}, \quad \text { if } t \in D_{2} .
$$

Using bounds (29) and (31), we have that, if $t \in D_{2}$,

$$
\begin{aligned}
|t-\mathrm{i} a|^{\nu-1} I(t) & =|t-\mathrm{i} a|^{\nu-1}\left(I_{1}(t)+I_{2}(t)\right) \\
& \leq 16 \mathrm{e}^{\max \{0, \rho-T\}}|t-\mathrm{i} a|^{\nu-1}\|\phi\|_{\nu}+C K_{2} K_{1}^{-1} C_{\rho}^{\nu}\|\phi\|_{\nu} \\
& \leq K\|\phi\|_{\nu},
\end{aligned}
$$

with $K=\max \left\{16 \mathrm{e}^{\max \{0, \rho-T\}}\left((\max \{T, \rho\})^{2}+a^{2}\right)^{(\nu-1) / 2}, C K_{2} K_{1}^{-1} C_{\rho}^{\nu}\right\}$. 
In an analogous way, one can see that

$$
|t+\mathrm{i} a|^{\nu-1} I(t) \leq K\|\phi\|_{\nu}, \quad \text { if } t \in D_{3} .
$$

Finally, using bounds (28), (32), and (33), we obtain that $I \in \mathcal{X}_{\nu-1}$ and $\|I\|_{\nu-1} \leq$ $(4+2 K)\|\phi\|_{v}$. Using (27), we have that $\mathcal{L}_{\alpha, c}(\phi) \in \mathcal{X}_{v-1}$, and moreover,

$$
\left\|\mathcal{L}_{\alpha, c}(\phi)\right\|_{\nu-1} \leq \mathrm{e}^{|c| \pi}(4+2 K)\|\phi\|_{\nu} .
$$

Proceeding analogously with $\mathcal{L}_{-\alpha,-c}$ and using that $\mathcal{S}=\left(\mathcal{L}_{\alpha, c} \circ \pi^{1}, \mathcal{L}_{-\alpha,-c} \circ \pi^{2}\right)$, we finish the proof of the lemma.

In Lemma 2.9 we enunciate the properties of $\mathcal{T}$ we will use in the sequel.

Lemma 2.9. The operator $\mathcal{T}: \mathcal{X}_{3} \rightarrow \mathcal{X}_{2}$ given by (21) is well defined and there exists a constant $K$, independent of $\delta$, such that

$$
\|\mathcal{T}(\phi)\|_{2} \leq K|\log \delta|\|\phi\|_{3}, \quad \text { for any } \phi \in \mathcal{X}_{3} .
$$

Proof. Let $t \in D_{2}$. First we claim that, by (1) and (2) of Lemma 2.7, and using that $|\phi(r)| \leq|r-\mathrm{i} a|^{-3}\|\phi\|_{3}$, for all $r \in[0, t]$,

$|\mathcal{T}(\phi)(t)| \leq K_{1}^{-2} K_{2}^{2}|t-\mathrm{i} a|^{-2}\|\phi\|_{3}\left|\int_{0}^{t} \frac{1}{|r-\mathrm{i} a|} d r\right| \leq C K_{1}^{-2} K_{2}^{2}|t-\mathrm{i} a|^{-2}\|\phi\|_{3}|\log \delta|$.

Hence,

$$
\left|\mathcal{T}(\phi)(t)(t-\mathrm{i} a)^{2}\right| \leq C K_{1}^{-2} K_{2}^{2}|\log \delta|\|\phi\|_{3}, \quad t \in D_{2},
$$

and analogously we prove that

$$
\left|\mathcal{T}(\phi)(t)(t+\mathrm{i} a)^{2}\right| \leq C K_{1}^{-2} K_{2}^{2}|\log \delta|\|\phi\|_{3}, \quad t \in D_{3} .
$$

Now we deal with the case $t \in D_{1}$. We define $t^{*}=-t T / \operatorname{Re} t$. We note that the segment $\overline{0 t^{*}} \subset D_{2}$ and $\overline{t^{*} t} \subset D_{1}$. We write

$$
\begin{aligned}
\mathcal{T}(\phi)(t) & =\frac{1}{\cosh ^{2} t} \int_{0}^{t *} \cosh ^{2} r \phi(r) d r+\frac{1}{\cosh ^{2} t} \int_{t^{*}}^{t} \cosh ^{2} r \phi(r) d r \\
& :=I_{1}(t)+I_{2}(t) .
\end{aligned}
$$

We begin by bounding $I_{1}(t)=\cosh ^{-2} t \cosh ^{2} t^{*} \mathcal{T}(\phi)\left(t^{*}\right)$. We note that, since $t \in D_{1}$, we have that $\operatorname{Re} t \geq T \geq 2 \log 2$ and hence $|\cosh t| \geq 1$. Moreover, $\left|\cosh t^{*}\right| \leq K_{2}\left|t^{*}-\mathrm{i} a\right|$, provided that $t^{*} \in D_{2}$. Using these properties and bound (34) for $t=t^{*}$, we get that

$$
\left|I_{1}(t)\right| \leq\left|\cosh ^{-2} t\right| \frac{\left|\cosh ^{2} t^{*}\right|}{\left|t^{*}-\mathrm{i} a\right|^{2}} C K_{1}^{-2} K_{2}^{2}|\log \delta|\|\phi\|_{3} \leq C K_{1}^{-2} K_{2}^{4}|\log \delta|\|\phi\|_{3} .
$$

Now we bound $I_{2}(t)$. We claim that

$$
\left|I_{2}(t)\right| \leq 16(1+a / T)\|\phi\|_{3} .
$$


Indeed, first we recall that $\mathrm{e}^{|\operatorname{Re} r|} / 4 \leq|\cosh r| \leq \mathrm{e}^{|\operatorname{Re} r|}$ and that $|\phi(r)| \leq\|\phi\|_{3}$ for all $r \in D_{1}$. Hence, since by definition of $t^{*}, \operatorname{Re} t^{*}=-T$. Parameterizing the integration path in $I_{2}$ by $\gamma(r)=t r+t^{*}(1-r)$, we have that

$$
\begin{aligned}
\left|I_{2}(t)\right| & \leq 16 \mathrm{e}^{-2|\operatorname{Re} t|}\left|t-t^{*}\right|\|\phi\|_{3} \int_{0}^{1} \mathrm{e}^{2(r \operatorname{Re} t-(1-r) T)} d r \\
& =16 \mathrm{e}^{-2(|\operatorname{Re} t|-T)} \frac{\left|t-t^{*}\right|}{\operatorname{Re} t+T}\left(\mathrm{e}^{2|\operatorname{Re} t+T|}-1\right)\|\phi\|_{3} .
\end{aligned}
$$

Finally, using that $\left|t-t^{*}\right|=|t||\operatorname{Re} t+T| /|\operatorname{Re} t|$, we get (38).

Hence, decomposition (36) and bounds (37) and (38) give us the following bound of $\mathcal{T}(\phi)(t)$ for all $t \in D_{1}$ :

$$
|\mathcal{T}(\phi)(t)| \leq C K_{1}^{-2} K_{2}^{4}|\log \delta|\|\phi\|_{3}+16(1+a / T)\|\phi\|_{3} \leq 2 C K_{1}^{-2} K_{2}^{4}|\log \delta|\|\phi\|_{3},
$$

if $\delta$ is small enough. Finally, bounds (34), (35), and (39) imply that

$$
\|\mathcal{T}(\phi)\|_{2} \leq 2\left(1+K_{2}^{2}\right) C K_{1}^{-2} K_{2}^{2}|\log \delta|\|\phi\|_{3},
$$

and the lemma is proved.

2.3.2. The Independent Term. Now we prove that the first approximation of $\varphi, \varphi_{1}=$ $\mathcal{L} \circ \mathcal{R}(0)$ (see (10) for the definition of the function $\mathcal{R}$ ), satisfies the properties enunciated in Proposition 2.5. Concretely, we will prove the following:

Lemma 2.10. The function $\mathcal{L} \circ \mathcal{R}(0) \in \mathcal{X}_{3} \times \mathcal{X}_{3} \times \mathcal{X}_{2}$, and moreover, there exists a constant $K$ independent of $\delta$ such that

$$
\|\mathcal{L} \circ \mathcal{R}(0)\| \leq K \delta^{p+4},
$$

where the norm $\|\cdot\|$ was defined in (17).

Proof. We note that $\mathcal{R}(0) \in \mathcal{X}_{0} \times \mathcal{X}_{0} \times \mathcal{X}_{0}$. This is due to the fact that $\delta z_{0}$ is bounded in $D_{\rho}$. Hence, by Remark 2.2, $\mathcal{R}(0) \in \mathcal{X}_{v} \times \mathcal{X}_{v} \times \mathcal{X}_{v}$ for any $v>0$, in particular for $v=3$.

First we claim that $D \mathcal{M}(0) \in \mathcal{X}_{4} \times \mathcal{X}_{4}$ (here $D$ denotes $\frac{d}{d t}$ ) and that there exists a constant $K_{\mathcal{M}}$ independent on $\delta$ such that

$$
\|\mathcal{M}(0)\|_{\times, 3} \leq K_{\mathcal{M}} \delta^{p+3}, \quad\|D \mathcal{M}(0)\|_{\times, 4} \leq K_{\mathcal{M}} \delta^{p+3} .
$$

(The norm $\|\cdot\|_{\times, 3}$ was defined in (18)). Indeed, we recall that by definition (11), $\mathcal{M}(0)(t)=\delta^{p} F\left(0,0, \delta z_{0}(t), \delta\right)$ and $F=\left(F_{1}, F_{2}\right)$ is an analytic function in $B^{3}\left(r_{0}\right) \times$ $B\left(\delta_{0}\right)$, such that $|F(0,0, z, \delta)| \leq C_{F}|(z, \delta)|^{3}$. Henceforth, for $t \in D_{\rho}$,

$$
|\mathcal{M}(0)(t)| \leq \delta^{p+3} C_{F}\left|\left(z_{0}(t), 1\right)\right|^{3},
$$

and since $z_{0} \in \mathcal{X}_{1}$ and $1 \in \mathcal{X}_{0} \subset \mathcal{X}_{1}$, we obtain $\|\mathcal{M}(0)\|_{\times, 3} \leq K_{\mathcal{M}} \delta^{p+3}$. 
Now let $(z, \delta) \in B\left(r_{0} / 2\right) \times B\left(\delta_{0}\right)$. We note that, if $\delta_{0}$ is small enough, then $z+$ $|(z, \delta)| \mathrm{e}^{\mathrm{i} \theta} / 2 \in B\left(r_{0}\right)$ for all $\theta \in[0,2 \pi]$. Hence, by Cauchy's theorem,

$$
\begin{aligned}
\left|\partial_{z} F(0,0, z, \delta)\right| & \leq \frac{1}{\pi|(z, \delta)|} \int_{0}^{2 \pi}\left|F\left(0,0, z+|(z, \delta)| \mathrm{e}^{\mathrm{i} \theta} / 2, \delta\right)\right| d \theta \\
& \leq \frac{27}{4} C_{F}|(z, \delta)|^{2} .
\end{aligned}
$$

As we pointed out in Section 2.2, we assume that $r_{0}$ is big enough to satisfy that $\delta z_{0}(t) \in$ $B\left(r_{0} / 2\right)$ for all $t \in D_{\rho}$. Therefore,

$$
|D \mathcal{M}(0)(t)|=\delta^{p+1}\left|\partial_{z} F\left(0,0, \delta z_{0}(t), \delta\right)\right|\left|D z_{0}(t)\right| \leq \frac{27}{4} C_{F} \delta^{p+3}\left|\left(z_{0}(t), 1\right)\right|^{2}\left|D z_{0}(t)\right|,
$$

and (40) is proved provided that $1 \in \mathcal{X}_{0} \subset \mathcal{X}_{1}, z_{0} \in \mathcal{X}_{1}$ and $D z_{0} \in \mathcal{X}_{2}$.

In addition we observe that, by integrating by parts the integral in the definition (20) of $\mathcal{L}_{\alpha, c}$, we have a more suitable expression for $\mathcal{L}_{\alpha, c}\left(\pi^{1} \mathcal{R}(0)\right)=\mathcal{L}_{\alpha, c}\left(\pi^{1} \mathcal{M}(0)\right)$ :

$$
\mathcal{L}_{\alpha, c}\left(\pi^{1} \mathcal{M}(0)\right)(t)=-\frac{\delta \mathrm{i}}{\alpha}\left[\pi^{1} \mathcal{M}(0)(t)-\mathcal{L}_{\alpha, c}\left(g_{1}\right)(t)\right],
$$

with

$$
g_{1}(t)=D\left(\pi^{1} \mathcal{M}(0)\right)(t)+z_{0}(t)(1+\mathrm{i} c) \cdot \pi^{1} \mathcal{M}(0)(t) .
$$

We obtain an analogous expression for $\mathcal{L}_{-\alpha,-c}\left(\pi^{2} \mathcal{M}(0)\right)$, and we conclude that

$$
\mathcal{S}(\mathcal{M}(0))=-\frac{\delta \mathrm{i}}{\alpha}\left(\begin{array}{rr}
1 & 0 \\
0 & -1
\end{array}\right)[\mathcal{M}(0)-\mathcal{S}(g)]
$$

with $g=\left(g_{1}, g_{2}\right)$ and $g_{2}(t)=D\left(\pi^{2} \mathcal{M}(0)\right)(t)+z_{0}(t)(1-\mathrm{i} c) \cdot \pi^{2} \mathcal{M}(0)(t)$. We note that by (40) and since $z_{0} \in \mathcal{X}_{1}, g \in \mathcal{X}_{4} \times \mathcal{X}_{4}$ and $\|g\|_{\times, 4} \leq K \delta^{p+3}$ for some constant $K$. Hence, by Lemma 2.8 and using again $(40), \mathcal{S} \circ \mathcal{M}(0) \in \mathcal{X}_{3} \times \mathcal{X}_{3}$ and

$$
\|\mathcal{S} \circ \mathcal{M}(0)\|_{\times, 3} \leq C_{0} \delta^{p+4} .
$$

Now we deal with $\mathcal{T} \circ \mathcal{N}$. As in the previous case, one can check that $\mathcal{N} \in \mathcal{X}_{3}$ and that there exists a constant $K_{\mathcal{N}}$ such that $\|\mathcal{N}\|_{3} \leq K_{\mathcal{N}} \delta^{p+3}$. Hence by Lemma 2.9, $\mathcal{T} \circ \mathcal{N} \in \mathcal{X}_{2}$ and

$$
\|\mathcal{T} \circ \mathcal{N}\|_{2} \leq K \delta^{p+3}|\log \delta|
$$

for some constant $K$ independent of $\delta$. Finally, by bounds (42) and (43) and definition (17) of the norm $\|\cdot\|$, we get the result.

2.3.3. End of the Proof of Proposition 2.5. To finish the proof of Proposition 2.5, we will need the following technical lemma:

Lemma 2.11 ([Ang93]). Let $E$ be a complex Banach space, and let $f: B_{r} \rightarrow B_{\theta r}$ be a holomorphic mapping, where $B_{\rho}=\{x \in E:\|x\|<\rho\}$.

If $\theta<1 / 2$, then $f_{\mid B_{\theta r}}$ is a contraction, and hence has a unique fixed point in $B_{\theta r}$. 
As we pointed out in Lemma 2.3, if $\varphi$ is a solution of

$$
\varphi=\mathcal{L} \circ \mathcal{R}(\varphi)
$$

then $\varphi$ is also a solution of system (13) satisfying that $\pi^{3} \varphi(0)=0$. Moreover, it is clear that, if $\varphi \in \mathcal{X}_{3} \times \mathcal{X}_{3} \times \mathcal{X}_{2}$, then $\varphi$ is bounded on $\bar{D}_{\rho}$.

We notice that, by Lemma $2.10, \varphi_{1}=\mathcal{L} \circ \mathcal{R}(0) \in \mathcal{X}_{3} \times \mathcal{X}_{3} \times \mathcal{X}_{2}$, and thus we denote

$$
R=8\left\|\varphi_{1}\right\| \leq 8 K \delta^{p+4}
$$

Lemma 2.12. We define $\mathcal{F}=\mathcal{L} \circ \mathcal{R}$ and $B(R)$ the closed ball of $\mathcal{X}_{3} \times \mathcal{X}_{3} \times \mathcal{X}_{2}$ centered at the origin of radius $R>0$.

The operator $\mathcal{F}: B(R) \rightarrow B(R / 4)$ is well defined and, moreover,

$$
\left\|\mathcal{F}(\varphi)-\varphi_{1}\right\| \leq K \delta^{p+2}|\log \delta|\|\varphi\|, \quad \text { for all } \varphi \in B(R) .
$$

Remark 2.13. We claim that Lemma 2.12 implies Proposition 2.5. Indeed, since the operator $\mathcal{F}$ is analytic in $B(R)$ and $\mathcal{F}(B(R)) \subset B(R / 4)$, Lemma 2.11 implies that the fixed point equation (44) has one solution $\varphi \in B(R)$. Moreover, taking $\varphi_{2}=\mathcal{F}(\varphi)-\varphi_{1}$, by (45), the statement (2) of Proposition 2.5 holds trivially. Item (1) of Proposition 2.5 is fulfilled by Lemma 2.10 .

Proof of Lemma 2.12. First we will prove bound (45). Let $\varphi \in B(R)$. Since $R=$ $8\left\|\varphi_{1}\right\| \leq 8 K \delta^{p+4}$, we have that $\|\varphi\| \leq C \delta^{p+4}$, with $C=8 K$.

We denote $\varphi=(\xi, \bar{\xi}, \eta)$, and we notice that

$$
\|(\xi, \bar{\xi})\|_{\times, 3} \leq\|\varphi\| \leq C \delta^{p+4}, \quad\|\eta\|_{2} \leq\|\varphi\| \delta^{-1}|\log \delta| \leq C \delta^{p+3}|\log \delta| .
$$

We also introduce

$$
\begin{aligned}
\Delta F(t) & =F\left(\delta \xi(t), \delta \bar{\xi}(t), \delta\left(z_{0}(t)+\eta(t)\right), \delta\right)-F\left(0,0, \delta z_{0}(t), \delta\right), \\
\Delta H(t) & =H\left(\delta \xi(t), \delta \bar{\xi}(t), \delta\left(z_{0}(t)+\eta(t)\right), \delta\right)-H\left(0,0, \delta z_{0}(t), \delta\right),
\end{aligned}
$$

and we observe that, since $\Delta F$ and $\Delta H$ are bounded in $D_{\rho}, \Delta F$ and $\Delta H$ belong to $\mathcal{X}_{0}$ and, by Remark $2.2, \Delta F \in \mathcal{X}_{4} \times \mathcal{X}_{4}$ and $\Delta H \in \mathcal{X}_{3}$.

Next we will bound $\|\Delta F\|_{\times, 4}$. Throughout this proof, we will denote $D=D_{x, y, z}$ the first derivative with respect to $(x, y, z)$. We note that, since $F$ is analytic on $B^{3}\left(r_{0}\right) \times$ $B\left(\delta_{0}\right)$, we have that, if $\delta_{0}$ is small enough and $(x, y, z, \delta) \in B^{3}\left(r_{0} / 2\right) \times B\left(\delta_{0}\right)$,

$$
|D F(x, y, z, \delta)| \leq C_{F}|(x, y, z, \delta)|^{2} .
$$

To check that (47) is straightforward, we use Cauchy's theorem in an analogous way as in (41). Then, using (47) and the mean's value theorem, we have that for all $t \in D_{\rho}$,

$$
\begin{aligned}
|\Delta F(t)| & \leq \delta \int_{0}^{1}\left|D F\left(\delta \xi(t) \lambda, \delta \bar{\xi}(t) \lambda, \delta\left(z_{0}(t)+\eta(t) \lambda\right), \delta\right)\right| d \lambda \cdot|\varphi(t)| \\
& \leq \delta^{3} C_{F}\left(\max \left\{|\xi(t)|,|\bar{\xi}(t)|,\left|z_{0}(t)\right|+|\eta(t)|, 1\right\}\right)^{2}|\varphi(t)| \\
& \leq 4 \delta^{3} C_{F}|\varphi(t)|\left(\max \left\{|\varphi(t)|,\left|z_{0}(t)\right|, 1\right\}\right)^{2} .
\end{aligned}
$$


Let $t \in D_{1}$. By (46) we have that $|\xi(t)|,|\bar{\xi}(t)| \leq\|\varphi\|$, and $|\eta(t)| \leq \delta^{-1}|\log \delta|\|\varphi\|$. Hence, $|\varphi(t)| \leq\|\varphi\| \delta^{-1}|\log \delta|$. Moreover, since $p>-2$ and $\|\varphi\| \leq C \delta^{p+4}$, we have that there exists a constant $M$ independent of $\delta$ such that

$$
\max \left\{|\varphi(t)|,\left|z_{0}(t)\right|, 1\right\} \leq M,
$$

if $\delta$ is small enough. Using these bounds to bound (48), we obtain

$$
|\Delta F(t)| \leq 4 \delta^{2}|\log \delta| C_{F}\|\varphi\| M^{2}, \quad t \in D_{1} .
$$

Now we fix $t \in D_{2}$. By (46) and since $|t-\mathrm{i} a| \geq \delta$, we have that

$$
\begin{aligned}
|\varphi(t)| & \leq\|\varphi\| \max \left\{|t-\mathrm{i} a|^{-3},|t-\mathrm{i} a|^{-2} \delta^{-1}|\log \delta|\right\} \\
& \leq \delta^{-1}|\log \delta|\|\varphi\||t-\mathrm{i} a|^{-2} .
\end{aligned}
$$

Using this bound and the fact that $\left|(t-\mathrm{i} a)^{-1} z_{0}(t)\right|$ is bounded in $D_{2}$, increasing $M$ if necessary, we have that

$$
\begin{aligned}
\max \left\{|\varphi(t)|,\left|z_{0}(t)\right|, 1\right\} & \leq \max \left\{\left\|z_{0}\right\|_{1}|t-\mathrm{i} a|^{-1}, \delta^{-1}|\log \delta|\|\varphi\||t-\mathrm{i} a|^{-2}, 1\right\} \\
& \leq M|t-\mathrm{i} a|^{-1},
\end{aligned}
$$

where we have used that $\delta$ is small enough and that $p>-2$. Therefore, bounding (48), we have that, for all $t \in D_{2}$,

$$
|t-\mathrm{i} a|^{4}|\Delta F(t)| \leq 4 \delta^{2}|\log \delta| C_{F}\|\varphi\| M^{2} .
$$

In the same way, one can check that

$$
|t+\mathrm{i} a|^{4}|\Delta F(t)| \leq 4 \delta^{2}|\log \delta| C_{F}\|\varphi\| M^{2}, \quad \text { if } t \in D_{3} .
$$

Thus using bounds (49), (51), and (52), we get

$$
\|\Delta F\|_{\times, 4} \leq 12 \delta^{2}|\log \delta| C_{F}\|\varphi\| M^{2} .
$$

We claim that $\xi \cdot \eta \in \mathcal{X}_{4}$ and

$$
\|\xi \cdot \eta\|_{4} \leq\|\varphi\| C \delta^{p+2}|\log \delta| .
$$

Indeed, it is clear that

$$
|\xi(t) \eta(t)| \leq\|\varphi\|^{2} \delta^{-1}|\log \delta| \leq C \delta^{p+3}|\log \delta|\|\varphi\|, \quad \text { if } t \in D_{1},
$$

and, since $|t \pm \mathrm{i} a| \geq \delta, \xi \in \mathcal{X}_{3}$, and $\eta \in \mathcal{X}_{2}$, we also have that

$$
\begin{aligned}
\left|(t-\mathrm{i} a)^{4} \xi(t) \eta(t)\right| & \leq|t-\mathrm{i} a|^{-1}\|\xi\|_{3}\|\eta\|_{2} & & \\
& \leq\|\varphi\| C \delta^{p+3}\left|\log \delta\left\|t-\left.\mathrm{i} a\right|^{-1} \leq\right\| \varphi \| C \delta^{p+2}\right| \log \delta \mid, & & \text { if } t \in D_{2} \\
\left|(t+\mathrm{i} a)^{4} \xi(t) \eta(t)\right| & \leq\|\varphi\| C \delta^{p+2}|\log \delta| & & \text { if } t \in D_{3} .
\end{aligned}
$$

Hence, the claim is proved. 
Analogously, we can also prove that $\|\bar{\xi} \cdot \eta\|_{4} \leq\|\varphi\| C \delta^{p+2}|\log \delta|$. Therefore, by definition (12) of $\mathcal{M}$ and bounds (53) and (54),

$$
\|\mathcal{M}(\varphi)-\mathcal{M}(0)\|_{\times, 4} \leq\|\varphi\| \delta^{p+2}|\log \delta|\left((1+|c|) C+12 C_{F} M^{2}\right),
$$

and Lemma 2.8 implies that $\mathcal{S}(\mathcal{M}(\varphi)-\mathcal{M}(0)) \in \mathcal{X}_{3} \times \mathcal{X}_{3}$ and

$$
\|\mathcal{S}(\mathcal{M}(\varphi)-\mathcal{M}(0))\|_{\times, 3} \leq K \delta^{p+2}|\log \delta|\|\varphi\|,
$$

for some constant $K$ independent of $\delta$.

Finally we deal with $\mathcal{T}(\mathcal{N}(\varphi)-\mathcal{N}(0))$. We observe that $\Delta H$ can be studied in the same way as $\Delta F$. Therefore, we can conclude that

$$
\|\Delta H\|_{3} \leq\|\Delta H\|_{4} \sup _{t \in D_{\rho}}|t-\mathrm{i} a|^{-1} \leq 12 \delta|\log \delta| C_{H}\|\varphi\| M^{2} .
$$

We also have that

$$
\|\xi \cdot \bar{\xi}\|_{3} \leq\|\varphi\| C \delta^{p+1}, \quad\left\|\eta^{2}\right\|_{3} \leq\|\varphi\| C \delta^{p+1}|\log \delta|^{2}
$$

and hence, there exists a constant $K$ such that $\|\mathcal{N}(\varphi)-\mathcal{N}(0)\|_{3} \leq\|\varphi\| K \delta^{p+1}|\log \delta|^{2}$. By Lemma 2.9, this implies that

$$
\|\mathcal{T}(\mathcal{N}(\varphi)-\mathcal{N}(0))\|_{2} \leq K \delta^{p+1}|\log \delta|^{2}\|\varphi\|,
$$

and therefore, by definition (17) of the norm $\|\cdot\|$, and using bounds (55) and (56) of $\|\mathcal{S}(\mathcal{M}(\varphi)-\mathcal{M}(0))\|_{\times, 3}$ and $\|\mathcal{T}(\mathcal{N}(\varphi)-\mathcal{N}(0))\|_{2}$, we get

$$
\begin{aligned}
\left\|\mathcal{F}(\varphi)-\varphi_{1}\right\| & =\|\mathcal{S}(\mathcal{M}(\varphi)-\mathcal{M}(0))\|_{\times, 3}+\delta|\log \delta|^{-1}\|\mathcal{T}(\mathcal{N}(\varphi)-\mathcal{N}(0))\|_{2} \\
& \leq K \delta^{p+2}|\log \delta|\|\varphi\|,
\end{aligned}
$$

and bound (45) is proved.

Now we are done since, by definition of $R$ and the previous bound, we have that

$$
\|\mathcal{F}(\varphi)\| \leq\left\|\varphi_{1}\right\|+\left\|\mathcal{F}(\varphi)-\varphi_{1}\right\| \leq \frac{R}{8}+K \delta^{p+2}|\log \delta| R<\frac{R}{4},
$$

provided that $p>-2$ and $\delta$ is small enough.

\section{Exponentially Small Splitting of the Heteroclinic Orbit}

Let $\Delta \varphi=\varphi^{\mathrm{u}}-\varphi^{\mathrm{s}}$ where $\varphi^{\mathrm{u}}$ and $\varphi^{\mathrm{s}}$ are the solutions of system (13) given in Proposition 2.5. These solutions are defined by $D_{\rho}^{\mathrm{u}, \mathrm{s}}$ respectively and they satisfy the equation

$$
\dot{\varphi}^{\mathrm{s}, \mathrm{u}}=A(t) \varphi^{\mathrm{s}, \mathrm{u}}+\mathcal{R}\left(\varphi^{\mathrm{s}, \mathrm{u}}\right) .
$$

Subtracting equations for $\varphi^{\mathrm{u}}$ and $\varphi^{\mathrm{s}}$, we obtain that $\Delta \varphi$ is defined in $D_{\rho}^{\mathrm{u}} \cap D_{\rho}^{\mathrm{s}}$ and it must satisfy the linear equation

$$
\dot{\zeta}=A(t) \zeta+B(t) \zeta
$$


whose coefficients depend on $\varphi^{\mathrm{s}, \mathrm{u}}$, and they are given by

$$
B(t)=\int_{0}^{1} D \mathcal{R}\left((1-\lambda) \varphi^{\mathrm{s}}(t)+\lambda \varphi^{\mathrm{u}}(t)\right) d \lambda .
$$

Here $D=D_{\xi, \bar{\xi}, \eta}$ denotes the first derivative with respect to $(\xi, \bar{\xi}, \eta)$. To prove Theorem 1.4 our strategy will be to exploit equation (57). The idea behind the proof is that if a solution of (57) is analytic and bounded in $D_{\rho}^{\mathrm{u}} \cap D_{\rho}^{\mathrm{s}}$, then it has to be exponentially small with respect to $\delta$ when $t \in \mathbb{R} \cap D_{\rho}^{\mathrm{u}} \cap D_{\rho}^{\mathrm{s}}$. This is clear if one considers equation (57) with $B \equiv 0$, due to the special form of the fundamental matrix $\Phi$ of $\dot{\zeta}=A(t) \zeta$ given in Lemma 2.1. The same idea can be adapted for the full equation (57) using the fact that $B$ is a small perturbation of $A$ for $\delta$ small enough.

Because we are not dealing in this paper with upper bounds of $\Delta \varphi$ but with an asymptotic expression of it, we need to decompose $\Delta \varphi=\Delta \varphi_{1}+\Delta \varphi_{2}$ in such a way that $\Delta \varphi_{1}$ is the dominant term. We observe that the obvious decomposition, suggested by Proposition 2.5, $\Delta \varphi_{1}=\varphi_{1}^{\mathrm{u}}-\varphi_{1}^{\mathrm{s}}$, with $\varphi_{1}^{\mathrm{s}, \mathrm{u}}=\mathcal{L}^{\mathrm{s}, \mathrm{u}} \circ \mathcal{R}(0)$, is not the most appropriate. The reason is that the third component of $\varphi_{1}^{\mathrm{s}, \mathrm{u}}$ is given by

$$
\pi^{3} \varphi_{1}^{\mathrm{s}, \mathrm{u}}(t)=\frac{1}{\cosh ^{2} t} \int_{0}^{t} \cosh ^{2} r \mathcal{N}(0) d r
$$

and then $\pi^{3}\left(\varphi_{1}^{\mathrm{u}}-\varphi_{1}^{\mathrm{s}}\right)(t)$ is identically zero.

For the full solution $\Delta \varphi$, this cancellation will no longer be true. As $\varphi^{\mathrm{u}, \mathrm{s}}$ verify (19), we can assume only that $\pi^{3} \Delta \varphi(0)=0$. This makes it necessary to look for another first approximation with a third component different from zero. On the other hand, we would like to keep the two first components of $\varphi_{1}^{\mathrm{u}}-\varphi_{1}^{\mathrm{s}}$ as the main term of $\pi^{1,2} \Delta \varphi$. We use the fact that $\Delta \varphi$ satisfies the homogeneous linear differential equation (57) and hence can be expressed as

$$
\Delta \varphi(t)=\Phi(t)\left[\Delta \varphi(0)+\int_{0}^{t} \Phi^{-1}(r) B(r) \Delta \varphi(r) d r\right]:=\mathcal{B}(\Delta \varphi)(t),
$$

with $\Phi$ given in Lemma 2.1. With a Gauss-Seidel type of argument, we can use the two first components of $\mathcal{B}(\Delta \varphi)(t)$ to compute the third one. So $\Delta \varphi$ can also be written as

$$
\Delta \varphi(t)=\Phi(t)\left[\Delta \varphi(0)+\left(\begin{array}{c}
\int_{0}^{t} \pi^{1,2}\left(\Phi^{-1}(r) B(r) \Delta \varphi(r)\right) d r \\
\int_{0}^{t} \pi^{3}\left[\Phi^{-1}(r) B(r)\left(\pi^{1,2} \mathcal{B}(\Delta \varphi)(r), \pi^{3} \Delta \varphi(r)\right)\right] d r
\end{array}\right)\right] .
$$

Once one has a suitable fixed point equation for $\Delta \varphi$, it is natural to define the dominant term as

$$
\Phi(t)\left[\Delta \varphi(0)+\left(\begin{array}{c}
0 \\
\int_{0}^{t} \pi^{3}\left[\Phi^{-1}(r) B(r)\left(\pi^{1,2}(\Phi(r) \Delta \varphi(0)), 0\right)\right] d r
\end{array}\right)\right] .
$$

Of course, there is no sense in using $\Delta \varphi(0)$ since we do not know it (in fact, our goal is to find an asymptotic expression for it); for this reason we use $\varphi_{1}^{\mathrm{u}}(0)-\varphi_{1}^{\mathrm{S}}(0)$ instead of $\Delta \varphi(0)$ in the expression of the dominant term (59). We notice that the two first components of this dominant term are (we recall that $\pi^{1,2} \mathcal{R}(0)=\mathcal{M}(0)$ )

$$
\pi^{1,2}\left(\Phi(t)\left(\varphi_{1}^{\mathrm{u}}(0)-\varphi_{1}^{\mathrm{s}}(0)\right)\right)=\mathcal{S}^{\mathrm{u}} \circ \mathcal{M}(0)(t)-\mathcal{S}^{\mathrm{s}} \circ \mathcal{M}(0)(t),
$$


where this equality is a consequence of the fact that the function $\varphi_{1}^{\mathrm{s}}-\varphi_{1}^{\mathrm{u}}$ is a solution of the homogeneous linear equation $\dot{\chi}=A(t) \chi$. In this way we take $\Delta \varphi_{1}=\left(\Delta \psi_{1}, \Delta \eta_{1}\right)$, with

$$
\begin{aligned}
\Delta \psi_{1}(t) & =\pi^{1,2}\left(\Phi(t)\left(\varphi_{1}^{\mathrm{u}}(0)-\varphi_{1}^{\mathrm{s}}(0)\right)\right)=\mathcal{S}^{\mathrm{u}} \circ \mathcal{M}(0)(t)-\mathcal{S}^{\mathrm{s}} \circ \mathcal{M}(0)(t) \\
& =\left(\begin{array}{l}
\mathrm{e}^{-\mathrm{i} \alpha t / \delta} \mathrm{e}^{\mathrm{i} c \log (\cosh t)} \cosh t \int_{-\infty}^{+\infty} \frac{1}{\cosh r} \mathrm{e}^{\mathrm{i} \alpha r / \delta} \mathrm{e}^{-\mathrm{i} c \log (\cosh r)} \pi^{1} \mathcal{M}(0)(r) d r \\
\mathrm{e}^{\mathrm{i} \alpha t / \delta} \mathrm{e}^{-\mathrm{i} c \log (\cosh t)} \cosh t \int_{-\infty}^{+\infty} \frac{1}{\cosh r} \mathrm{e}^{-\mathrm{i} \alpha r / \delta} \mathrm{e}^{\mathrm{i} c \log (\cosh r)} \pi^{2} \mathcal{M}(0)(r) d r
\end{array}\right), \\
\Delta \eta_{1}(t) & =\frac{1}{\cosh ^{2} t} \int_{0}^{t} \cosh ^{2} r \pi^{3}\left[B(r)\left(\Delta \psi_{1}(r), 0\right)\right] d r
\end{aligned}
$$

We denote

$$
\begin{aligned}
& c_{1}^{0}=\pi^{1}\left(\varphi_{1}^{\mathrm{u}}(0)-\varphi_{1}^{\mathrm{s}}(0)\right)=\int_{-\infty}^{+\infty} \frac{1}{\cosh r} \mathrm{e}^{\mathrm{i} \alpha r / \delta} \mathrm{e}^{-\mathrm{i} c \log (\cosh r)} \pi^{1} \mathcal{M}(0)(r) d r, \\
& c_{2}^{0}=\pi^{2}\left(\varphi_{1}^{\mathrm{u}}(0)-\varphi_{1}^{\mathrm{s}}(0)\right)=\int_{-\infty}^{+\infty} \frac{1}{\cosh r} \mathrm{e}^{-\mathrm{i} \alpha r / \delta} \mathrm{e}^{\mathrm{i} c \log (\cosh r)} \pi^{2} \mathcal{M}(0)(r) d r
\end{aligned}
$$

and therefore

$$
\Delta \psi_{1}(t)=\left(\begin{array}{l}
\mathrm{e}^{-\mathrm{i} \alpha t / \delta} \mathrm{e}^{\mathrm{i} c \log (\cosh t)} \cosh t c_{1}^{0} \\
\mathrm{e}^{\mathrm{i} \alpha t / \delta} \mathrm{e}^{-\mathrm{i} c \log (\cosh t)} \cosh t c_{2}^{0}
\end{array}\right)
$$

Lemma 3.1. The constants $c_{1}^{0}$ and $c_{2}^{0}$ satisfy that $c_{1}^{0}=\overline{c_{2}^{0}}$ and that

$$
\left|c_{1}^{0}\right|=\left|c_{2}^{0}\right| \leq K \delta^{p} \mathrm{e}^{-\alpha a / \delta}
$$

We notice that this implies that $\left|\Delta \psi_{1}(0)\right| \leq K \delta^{p} \mathrm{e}^{-\alpha a / \delta}$.

Proof. The equality $c_{1}^{0}=\overline{c_{2}^{0}}$ comes from definition (61) of $c_{1}^{0}, c_{2}^{0}$ and from the fact that $\pi^{1} \mathcal{R}(0)=\overline{\pi^{2} \mathcal{R}(0)}$ (see definition (10) of $\mathcal{R}$ ). Moreover, since by Lemma 2.10, $\left\|\varphi_{1}^{\mathrm{s}, \mathrm{u}}\right\| \leq K \delta^{p+4}$, we have that $\left|\pi^{1} \Delta \psi_{1}(t)(t-\mathrm{i} a)^{3}\right| \leq\left\|\varphi_{1}^{\mathrm{u}}\right\|+\left\|\varphi_{1}^{\mathrm{s}}\right\| \leq K \delta^{p+4}$ for all $t \in D_{\rho}^{\mathrm{u}} \cap D_{\rho}^{\mathrm{s}}$. Then, since

$$
\left|\pi^{1} \Delta \psi_{1}(\mathrm{i}(a-\delta))\right|=\left|\mathrm{e}^{\alpha(a-\delta) / \delta} \mathrm{e}^{\mathrm{i} c \log (\cosh \mathrm{i}(a-\delta))} \cosh \mathrm{i}(a-\delta) c_{1}^{0}\right| \leq K \delta^{p+1},
$$

and $a=\pi / 2$, we have that $\left|c_{1}^{0}\right| \leq K \delta^{p} \mathrm{e}^{-\alpha a / \delta}$. Here we have used the fact that, taking the main determination of the $\operatorname{logarithm},\left|\mathrm{e}^{-\mathrm{i} c \log (\cosh \mathrm{i}(a-\delta))}\right| \leq \mathrm{e}^{|c| \pi / 2}$ and that by statement (2) of Lemma 2.7, $|\cosh s| \geq K_{1}|s-\mathrm{i} a| \geq K_{1} \delta$.

Remark 3.2. In fact, $c_{1}^{0}$ and $c_{2}^{0}$ will be computed more explicitly in Section 3.4 to get the asymptotic expression for $\Delta \varphi$ in Proposition 3.4.

The remaining part of this section is devoted to proving Theorem 1.4, which will be a direct consequence of Proposition 3.3 and Proposition 3.4, enunciated below. We postpone their proofs to the following subsections. 
In order to see that $\Delta \varphi(0)$ is given asymptotically by $\Delta \varphi_{1}(0)$, our next goal is to obtain an exponentially small bound for the difference $\Delta \varphi(0)-\Delta \varphi_{1}(0)$.

Proposition 3.3. If $p>-2$,

$$
\Delta \varphi(0)=\Delta \varphi_{1}(0)+O\left(\delta^{2 p+2}|\log \delta|\right) \mathrm{e}^{-|\alpha| \pi /(2 \delta)} .
$$

Finally, we check that $\Delta \varphi_{1}$ is actually the dominant term of $\Delta \varphi$.

Proposition 3.4. If $p>-2$ and $\delta$ is small enough,

$$
\begin{aligned}
\pi^{1,2} \Delta \varphi_{1}(0)= & 2 \pi \delta^{p} \mathrm{e}^{-\pi|\alpha| /(2 \delta)} \sum_{n \geq 3} \alpha^{n}\left(\begin{array}{c}
\mathrm{i}^{n}\left(\frac{|\alpha|}{\delta}\right)^{\mathrm{i} c} \frac{m_{n}}{\Gamma(n+1+\mathrm{i} c)} \\
(-\mathrm{i})^{n}\left(\frac{|\alpha|}{\delta}\right)^{-\mathrm{i} c} \frac{\overline{m_{n}}}{\Gamma(n+1-\mathrm{i} c)}
\end{array}\right) \\
& +O\left(\delta^{p+1}\right) \mathrm{e}^{-\pi|\alpha| /(2 \delta)},
\end{aligned}
$$

where the coefficients $m_{n}$ were defined in Theorem 1.4.

End of the proof of Theorem 1.4. We point out that Proposition 3.3 and Proposition 3.4 imply, trivially, Theorem 1.4 if we observe that

$$
\pi^{1} \Delta \sigma_{1}(0)+\mathrm{i} \pi^{2} \Delta \sigma_{1}(0)=\pi^{1} \Delta \varphi_{1}(0),
$$

and

$$
\begin{aligned}
\pi^{1} \Delta \varphi_{1}(0)= & 2 \pi \delta^{p} \mathrm{e}^{-\pi|\alpha| /(2 \delta)}\left(\frac{|\alpha|}{\delta}\right)^{\mathrm{i} c}(\alpha \mathrm{i})^{-\mathrm{i} c} \sum_{n \geq 3} \frac{(\alpha \mathrm{i})^{n+\mathrm{i} c} m_{n}}{\Gamma(n+1+\mathrm{i} c)} \\
& +O\left(\delta^{p+1}\right) \mathrm{e}^{-\pi|\alpha| /(2 \delta)} \\
= & 2 \pi \mathrm{e}^{c \pi / 2} \hat{m}(\mathrm{i} \alpha) \delta^{p} \mathrm{e}^{-\pi|\alpha| /(2 \delta)} \mathrm{e}^{-\mathrm{i} c \log \delta}+O\left(\delta^{p+1}\right) \mathrm{e}^{-\pi|\alpha| /(2 \delta)}
\end{aligned}
$$

The remaining part of the paper is devoted to proving these results.

Since we are interested only in computing $\Delta \varphi(0)$, it will be enough to study the behavior of $\Delta \varphi(s)$ for $s=\mathrm{i} t$, purely imaginary. We restrict our definition domain to

$$
E:=\{\mathrm{i} t \in \mathrm{i} \mathbb{R}:|t| \leq a-\delta\} \subset D_{\rho}^{\mathrm{s}} \cap D_{\rho}^{\mathrm{u}} .
$$

Obviously, $\Delta \varphi$ and $\Delta \varphi_{1}$ are both defined in $E$.

\subsection{The Solutions of Equation (57)}

In this subsection we find a suitable expression of $\Delta \varphi$ by means of a linear operator. 
Since $\varphi^{\mathrm{s}, \mathrm{u}} \in \mathcal{X}_{3}^{\mathrm{s}, \mathrm{u}} \times \mathcal{X}_{3}^{\mathrm{s}, \mathrm{u}} \times \mathcal{X}_{2}^{\mathrm{s}, \mathrm{u}}$, it is natural to consider the following normed space $\mathcal{Y}$ where $\Delta \varphi=\varphi^{\mathrm{u}}-\varphi^{\mathrm{s}}$ belongs to

$$
\begin{aligned}
\mathcal{Y}= & \left\{f: E \rightarrow \mathbb{C}^{3}: f\right. \text { is continuous } \\
\|f\|_{\mathcal{Y}}:= & \sup _{\mathrm{i} t \in E}\left|\pi^{1} f(\mathrm{i} t) \cos ^{3} t\right|+\sup _{\mathrm{i} t \in E}\left|\pi^{2} f(\mathrm{i} t) \cos ^{3} t\right| \\
& \left.+\frac{\delta}{|\log \delta|} \sup _{\mathrm{i} t \in E}\left|\pi^{3} f(\mathrm{i} t) \cos ^{2} t\right|<\infty\right\} .
\end{aligned}
$$

We introduce the operator $\mathcal{F}_{0}(f)=(\mathcal{G}(f), \mathcal{H}(f))$, where

$$
\begin{aligned}
& \mathcal{G}(f)(\mathrm{i} t)=\left(\begin{array}{l}
\mathrm{ie}^{\alpha t / \delta} \mathrm{e}^{\mathrm{i} c \log (\cos t)} \cos t \int_{a-\delta}^{t} \frac{\mathrm{e}^{-\alpha r / \delta} \mathrm{e}^{-\mathrm{i} c \log (\cos r)}}{\cos r} \pi^{1}(B(\mathrm{i} r) f(\mathrm{i} r)) d r \\
\mathrm{i} \mathrm{e}^{-\alpha t / \delta} \mathrm{e}^{-\mathrm{i} c \log (\cos t)} \cos t \int_{-(a-\delta)}^{t} \frac{\mathrm{e}^{\alpha r / \delta} \mathrm{e}^{\mathrm{i} c \log (\cos r)}}{\cos r} \pi^{2}(B(\mathrm{i} r) f(\mathrm{i} r)) d r
\end{array}\right), \\
& \mathcal{H}(f)(\mathrm{i} t)=\frac{\mathrm{i}}{\cos ^{2} t} \int_{0}^{t} \cos ^{2} r \pi^{3}\left(B(\mathrm{i} r)\left(\mathcal{G}(f)(\mathrm{i} r), \pi^{3} f(\mathrm{i} r)\right)\right) d r .
\end{aligned}
$$

In order to shorten the notation, we also define for all $k_{1}, k_{2} \in \mathbb{C}$,

$$
\begin{aligned}
& I\left(k_{1}, k_{2}\right)(t) \\
& =\left(\begin{array}{l}
k_{1} \mathrm{e}^{\alpha t / \delta} \mathrm{e}^{\mathrm{i} c \log (\cos t)} \cos t \\
k_{2} \mathrm{e}^{-\alpha t / \delta} \mathrm{e}^{-\mathrm{i} c \log (\cos t)} \cos t \\
\frac{\mathrm{i}}{\cos ^{2} t} \int_{0}^{t} \cos ^{3} r \pi^{3}\left(B(\mathrm{i} r)\left(k_{1} \mathrm{e}^{\alpha r / \delta} \mathrm{e}^{\mathrm{i} c \log (\cos r)}, k_{2} \mathrm{e}^{\alpha r / \delta} \mathrm{e}^{-\mathrm{i} c \log (\cos r)}, 0\right)\right) d r
\end{array}\right) .
\end{aligned}
$$

Lemma 3.5. $\Delta \varphi \in \mathcal{Y}$ and $\|\Delta \varphi\|_{\mathcal{Y}} \leq K \delta^{p+4}$. Moreover, there exist $c_{1}, c_{2} \in \mathbb{C}$ such that

$$
\Delta \varphi(\mathrm{i} t)=I\left(c_{1}, c_{2}\right)(t)+\mathcal{F}_{0}(\Delta \varphi)(\mathrm{i} t),
$$

and $\left|c_{1}\right|,\left|c_{2}\right| \leq K \delta^{p} \mathrm{e}^{-\alpha a / \delta}$

Proof. Since $\varphi^{\mathrm{s}, \mathrm{u}} \in \mathcal{X}_{3}^{\mathrm{s}, \mathrm{u}} \times \mathcal{X}_{3}^{\mathrm{s}, \mathrm{u}} \times \mathcal{X}_{2}^{\mathrm{s}, \mathrm{u}}, \Delta \varphi \in \mathcal{Y}$ obviously. Moreover, by Proposition 2.5, $\|\Delta \varphi\| \mathcal{Y} \leq\left\|\varphi^{\mathrm{u}}\right\| \mathcal{Y}+\left\|\varphi^{\mathrm{s}}\right\| \mathcal{Y} \leq K \delta^{p+4}$.

Now we check (66). Since $\Delta \varphi$ is a solution of the linear homogeneous equation (57), it can be written as

$$
\begin{aligned}
& \Delta \varphi(s) \\
& =\left(\begin{array}{l}
\mathrm{e}^{-\mathrm{i} \alpha s / \delta} \mathrm{e}^{\mathrm{i} c \log (\cosh s)} \cosh t\left[c_{1}+\int_{s_{1}}^{s} \frac{\mathrm{e}^{\mathrm{i} \alpha r / \delta} \mathrm{e}^{-\mathrm{i} c \log (\cosh r)}}{\cosh r} \pi^{1}(B(r) \Delta \varphi(r)) d r\right] \\
\mathrm{e}^{\mathrm{i} \alpha s / \delta} \mathrm{e}^{-\mathrm{i} c \log (\cosh s)} \cosh t\left[c_{2}+\int_{s_{2}}^{\mathrm{e}^{-\mathrm{i} \alpha r / \delta} \mathrm{e}^{\mathrm{i} c \log (\cosh r)}}\right. \\
\left.\frac{1}{\cosh r} \pi^{2}(B(r) \Delta \varphi(r)) d r\right]
\end{array}\right],
\end{aligned}
$$


for suitable $c_{1}, c_{2}, c_{3} \in \mathbb{C}$. We take $s_{3}=0, s_{1}=\mathrm{i}(a-\delta)$ and $s_{2}=-\mathrm{i}(a-\delta)$. We observe that, since we have chosen $\pi^{3} \varphi^{\mathrm{s}}(0)=\pi^{3} \varphi^{\mathrm{u}}(0)=0$, we have that $\pi^{3} \Delta \varphi(0)=0$ and then $c_{3}=0$. We perform the change of variables $r=\mathrm{i} u$ in all the integrals of (67) and we obtain, taking $s=\mathrm{i} t$ in (67),

$$
\begin{aligned}
\Delta \varphi(\mathrm{i} t)= & \left(\begin{array}{l}
c_{1} \mathrm{e}^{\alpha t / \delta} \mathrm{e}^{\mathrm{i} c \log (\cos t)} \cos t \\
c_{2} \mathrm{e}^{-\alpha t / \delta} \mathrm{e}^{-\mathrm{i} c \log (\cos t)} \cos t \\
0
\end{array}\right) \\
& +\left(\begin{array}{l}
\mathcal{G}(\Delta \varphi)(\mathrm{i} t) \\
\frac{\mathrm{i}}{\cos ^{2} t} \int_{0}^{t} \cos ^{2} u \pi^{3}(B(\mathrm{i} u) \Delta \varphi(\mathrm{i} u)) d u
\end{array}\right) .
\end{aligned}
$$

Substituting the two first components of $\Delta \varphi, \pi^{1,2} \Delta \varphi$, by the right-hand side of (68), which is $\left(c_{1} \mathrm{e}^{\alpha t / \delta} \mathrm{e}^{\mathrm{i} c \log \cos t} \cos t, c_{2} \mathrm{e}^{-\alpha t / \delta} \mathrm{e}^{-\mathrm{i} c \log \cos t} \cos t\right)^{\mathrm{T}}+\mathcal{G}(\Delta \varphi)$, in the third component of the expression (68) of $\Delta \varphi(\mathrm{i} t)$, we get the form (66) stated in the lemma.

Now we bound $\left|c_{1}\right|,\left|c_{2}\right|$. Since $\Delta \varphi \in \mathcal{Y}$, we have that $\left|\pi^{1}(\Delta \varphi(\mathrm{i} t)) \cos ^{3} t\right| \leq\|\Delta \varphi\|_{\mathcal{Y}}$. Then, since $s_{1}=\mathrm{i}(a-\delta)$,

$$
\left|\pi^{1}\left(\Delta \varphi\left(s_{1}\right)\right) \cos ^{3}(a-\delta)\right|=\left|\mathrm{e}^{\alpha(a-\delta) / \delta} \mathrm{e}^{\mathrm{i} c \log (\cos (a-\delta))} \cos ^{4}(a-\delta) c_{1}\right| \leq\|\Delta \varphi\|_{\mathcal{Y}},
$$

and thus, since $a=\pi / 2,\left|c_{1}\right| \leq\|\Delta \varphi\|_{\mathcal{Y}} \mathrm{e}^{-\alpha(a-\delta) / \delta}\left|\mathrm{e}^{-\mathrm{i} c \log (\cos (a-\delta))}\right| \delta^{-4}$. In the same way, one can check that $\left|c_{2}\right| \leq\|\Delta \varphi\| \mathcal{Y} \mathrm{e}^{-\alpha(a-\delta) / \delta}\left|\mathrm{e}^{\mathrm{i} c \log (\cos (a-\delta))}\right| \delta^{-4}$.

Finally we observe that $\left|\mathrm{e}^{ \pm \mathrm{i} c \log (\cos (a-\delta))}\right| \leq \mathrm{e}^{|c| \pi / 2}$, taking the main determination of the logarithm. Therefore, since $\|\Delta \varphi\|_{\mathcal{Y}} \leq K \delta^{p+4}$, the lemma is proved.

\subsection{The Equation for $\Delta \varphi_{2}:=\Delta \varphi-\Delta \varphi_{1}$}

We denote $\Delta \varphi_{2}=\Delta \varphi-\Delta \varphi_{1}$ and we decompose $\Delta \varphi=(\Delta \psi, \Delta \eta)$ with $\Delta \psi=\pi^{1,2} \Delta \varphi$ and $\Delta \eta=\pi^{3} \Delta \varphi$. Analogously, we will write $\Delta \varphi_{2}=\left(\Delta \psi_{2}, \Delta \eta_{2}\right)$.

We recall that by (62), (60), and definition (65) of $I$, we have that $\Delta \varphi_{1}=I\left(c_{1}^{0}, c_{2}^{0}\right)$, and by (63) in Lemma 3.1, $\left|c_{1}^{0}\right|=\left|c_{2}^{0}\right| \leq K \delta^{p} \mathrm{e}^{-\alpha a / \delta}$.

The following lemma expresses $\Delta \varphi_{2}$ in a more appropriate way in terms of $I\left(k_{1}, k_{2}\right)$ and of the linear operator $\mathcal{F}_{0}$. We also provide useful bounds of $k_{1}$ and $k_{2}$.

Lemma 3.6. $\Delta \varphi_{2}=\Delta \varphi-\Delta \varphi_{1}$ satisfies the fixed point equation given by

$$
\Delta \varphi_{2}(\mathrm{i} t)=I\left(c_{1}-c_{1}^{0}, c_{2}-c_{2}^{0}\right)(t)+\mathcal{F}_{0}\left(\Delta \varphi_{1}\right)(\mathrm{i} t)+\mathcal{F}_{0}\left(\Delta \varphi_{2}\right)(\mathrm{i} t) .
$$

Moreover, there exists a constant $K$ such that

$$
\left|c_{1}-c_{1}^{0}\right|,\left|c_{2}-c_{2}^{0}\right| \leq K \delta^{2 p+2} \mathrm{e}^{-\alpha a / \delta} .
$$

Proof. To prove (69), we only have to take into account expression (66) of $\Delta \varphi$ in Lemma 3.5, that $\Delta \varphi_{1}(\mathrm{i} t)=I\left(c_{1}^{0}, c_{2}^{0}\right)(t)$ and the fact that $\mathcal{F}_{0}$ is linear.

Now we deal with (70). As we pointed out in Remark 3.1, $\Delta \psi_{1}=\pi^{1,2}\left(\varphi_{1}^{\mathrm{u}}-\varphi_{1}^{\mathrm{s}}\right)$, thus we have that $\Delta \psi_{2}=\pi^{1,2}\left(\varphi_{2}^{\mathrm{u}}-\varphi_{2}^{\mathrm{s}}\right)$, where we recall that $\varphi_{2}^{\mathrm{s}, \mathrm{u}}$ were defined in Proposition 2.5. Then, it is clear that, by item (2) in Proposition 2.5,

$$
\left|\pi^{1,2} \Delta \psi_{2}(\mathrm{i} t) \cos ^{3} t\right| \leq\left\|\varphi_{2}^{\mathrm{s}}\right\|+\left\|\varphi_{2}^{\mathrm{u}}\right\| \leq K \delta^{2 p+6} .
$$


In addition, we observe that, taking into account expression (62) of $\Delta \psi_{1}$,

$$
\begin{aligned}
& c_{1}^{0}=\pi^{1} \Delta \psi_{1}(\mathrm{i}(a-\delta)) \mathrm{e}^{-\alpha(a-\delta)} \mathrm{e}^{-\mathrm{i} c \log (\cos (a-\delta))} \cos ^{-1}(a-\delta), \\
& c_{2}^{0}=\pi^{2} \Delta \psi_{1}(-\mathrm{i}(a-\delta)) \mathrm{e}^{-\alpha(a-\delta)} \mathrm{e}^{\mathrm{i} c \log (\cos (a-\delta))} \cos ^{-1}(a-\delta)
\end{aligned}
$$

(an analogous formula can be deduced for $c_{1}$ and $c_{2}$ using $\Delta \psi$ instead of $\Delta \psi_{1}$ ). Therefore,

$$
\begin{aligned}
c_{1}-c_{1}^{0}= & \mathrm{e}^{-\alpha(a-\delta) / \delta} \mathrm{e}^{-\mathrm{i} c \log (\cos (a-\delta))} \cos ^{-1}(a-\delta) \pi^{1}\left(\Delta \psi(\mathrm{i}(a-\delta))-\Delta \psi_{1}(\mathrm{i}(a-\delta))\right) \\
= & \mathrm{e}^{-\alpha(a-\delta) / \delta} \mathrm{e}^{-\mathrm{i} c \log (\cos (a-\delta))} \cos ^{-1}(a-\delta) \pi^{1} \Delta \psi_{2}(\mathrm{i}(a-\delta)), \\
c_{2}-c_{2}^{0}= & \mathrm{e}^{-\alpha(a-\delta) / \delta} \mathrm{e}^{\mathrm{i} c \log (\cos (a-\delta))} \cos ^{-1}(a-\delta) \pi^{2}(\Delta \psi(-\mathrm{i}(a-\delta)) \\
& \left.-\Delta \psi_{1}(-\mathrm{i}(a-\delta))\right) \\
= & \mathrm{e}^{-\alpha(a-\delta) / \delta} \mathrm{e}^{\mathrm{i} c \log (\cos (a-\delta))} \cos ^{-1}(a-\delta) \pi^{2} \Delta \psi_{2}(-\mathrm{i}(a-\delta)) .
\end{aligned}
$$

Hence, using (71) to bound $\pi^{1,2} \Delta \psi_{2}( \pm \mathrm{i}(a-\delta))$ in expressions (72) and (73), we obtain

$$
\left|c_{1}-c_{1}^{0}\right|,\left|c_{2}-c_{2}^{0}\right| \leq K \delta^{2 p+2} \mathrm{e}^{-\alpha a / \delta} .
$$

\subsection{Exponential Smallness of $\Delta \varphi_{2}$}

Let us introduce the functional spaces

$$
\begin{aligned}
& \mathcal{Z}_{1}=\left\{f: E \rightarrow \mathbb{C}: f \text { is continuous and } \sup _{\mathrm{i} t \in E}\left|\mathrm{e}^{\alpha(a-|t|) / \delta} \cos ^{-1} t f(\mathrm{i} t)\right|<+\infty\right\}, \\
& \mathcal{Z}_{2}=\left\{f: E \rightarrow \mathbb{C}: f \text { is continuous and } \sup _{\mathrm{i} t \in E}\left|\mathrm{e}^{\alpha(a-|t|) / \delta} \cos t f(\mathrm{i} t)\right|<+\infty\right\} .
\end{aligned}
$$

We endow $\mathcal{Z}_{1}$ and $\mathcal{Z}_{2}$ with the norms

$$
\begin{aligned}
& \left\|f_{1}\right\|_{\mathcal{Z}_{1}}=\sup _{\mathrm{i} t \in E}\left|\mathrm{e}^{\alpha(a-|t|) / \delta} \frac{1}{\cos t} f_{1}(\mathrm{i} t)\right|, \\
& \left\|f_{2}\right\|_{\mathcal{Z}_{2}}=\sup _{\mathrm{i} t \in E}\left|\mathrm{e}^{\alpha(a-|t|) / \delta} \cos t f_{2}(\mathrm{i} t)\right| .
\end{aligned}
$$

We also consider the product space $\mathcal{Z}=\mathcal{Z}_{1} \times \mathcal{Z}_{1} \times \mathcal{Z}_{2}$ with the norm

$$
\|g\|_{\mathcal{Z}}=\left\|\pi^{1} g\right\|_{\mathcal{Z}_{1}}+\left\|\pi^{2} g\right\|_{\mathcal{Z}_{1}}+|\log \delta|^{-1} \delta^{-p-4}\left\|\pi^{3} g\right\|_{\mathcal{Z}_{2}}
$$

We notice that, if $g \in \mathcal{Z}$,

$$
\left|\pi^{1} g(0)\right|,\left|\pi^{2} g(0)\right| \leq\|g\|_{\mathcal{Z}} \mathrm{e}^{-\alpha a / \delta} \quad \text { and } \quad\left|\pi^{3} g(0)\right| \leq\|g\|_{\mathcal{Z}} \delta^{p+4}|\log \delta| \mathrm{e}^{-\alpha a / \delta} .
$$

Hence, in order to prove Proposition 3.3, we have to check that $\Delta \varphi_{2}$ expressed as (69) belongs to $\mathcal{Z}$ and $\left\|\Delta \varphi_{2}\right\|_{\mathcal{Z}} \leq K \delta^{2 p+2}$. Our method to prove these properties will be to check that both $I\left(c_{1}-c_{1}^{0}, c_{2}-c_{2}^{0}\right)$ and $\mathcal{F}_{0}\left(\Delta \varphi_{1}\right)$ belong to $\mathcal{Z}$ and that the operator Id $-\mathcal{F}_{0}$ is invertible in $\mathcal{Z}$. Moreover, since we have to bound $\left\|\Delta \varphi_{2}\right\|_{\mathcal{Z}}$, we also provide bounds of $\left\|\mathcal{F}_{0}\right\|_{\mathcal{Z}}:=\max \left\{\left\|\mathcal{F}_{0}(f)\right\|,\|f\|_{\mathcal{Z}}=1\right\}$.

All the properties related to the operator $\mathcal{F}_{0}$ are enunciated and proved below in Section 3.3.1. The remaining part of the proof of the exponential smallness of $\Delta \varphi_{2}$ is given in Section 3.3.2. 
3.3.1. The Operator $\mathcal{F}_{0}$. First we state a technical lemma related to matrix $B$ defined in (58).

Lemma 3.7. The matrix $B=\left(b_{i, j}\right)$ satisfies that there exists a constant $K$ independent of $\delta$ such that for all $i, j \in\{1,2,3\}$,

$$
\sup _{\mathrm{i} t \in E}\left|\cos ^{2} t b_{i, j}(\mathrm{i} t)\right| \leq K \delta^{p+3}|\log \delta|, \quad \text { for } \mathrm{i} t \in E .
$$

Proof. We denote $\psi_{\lambda}=\varphi^{\mathrm{s}}+\lambda \Delta \varphi=\left(\xi_{\lambda}, \bar{\xi}_{\lambda}, \eta_{\lambda}\right)$. It is clear that, by Lemma 3.5 and Proposition 2.5, $\psi_{\lambda} \in \mathcal{Y}$ and $\left\|\psi_{\lambda}\right\| \mathcal{Y} \leq K \delta^{p+4}$.

Let $b_{i, j}(t), i, j=1,2,3$ be the coefficients of the matrix $B$ defined in (58). We have that there exists a constant $C$ such that for all $\mathrm{i} t \in E$,

$$
\begin{aligned}
\left|b_{1, j}(\mathrm{i} t)\right| \leq & \left|\pi^{1} D \mathcal{R}\left(\psi_{\lambda}(\mathrm{i} t)\right)\right| \\
\leq & \left|\delta^{p+1} D F_{1}\left(\delta \xi_{\lambda}(\mathrm{i} t), \delta \bar{\xi}_{\lambda}(\mathrm{i} t), \delta\left(z_{0}(\mathrm{i} t)+\eta_{\lambda}(\mathrm{i} t)\right), \delta\right)\right| \\
& +C\left|\psi_{\lambda}(\mathrm{i} t)\right| .
\end{aligned}
$$

We recall that $\mathcal{R}$ was defined in (10), $|\cdot|$ denotes the maximum norm in $\mathbb{C}^{3}$, and $D=D_{\xi, \bar{\xi}, \eta}$ denotes the first derivative with respect to $(\xi, \bar{\xi}, \eta)$. Then, using bound (47) of $D F$, that $\left|\cos ^{2} t \eta_{\lambda}(\mathrm{i} t)\right| \leq K \delta^{p+3}|\log \delta|$ and that $\left|\cos ^{2} t \xi_{\lambda}(\mathrm{i} t)\right|,\left|\cos ^{2} t \bar{\xi}_{\lambda}(\mathrm{i} t)\right| \leq$ $K \delta^{p+4}(\cos t)^{-1} \leq K \delta^{p+3}$, we can bound (75), obtaining

$$
\left|\cos ^{2} t b_{i, j}(\mathrm{i} t)\right| \leq 4 \delta^{p+3} C_{F}\left|\cos ^{2} t\right|\left(\max \left\{\psi_{\lambda}(\mathrm{i} t)|,| z_{0}(t) \mid, 1\right\}\right)^{2}+K \delta^{p+3}|\log \delta|,
$$

if $t \in D_{2} \cap E$. Finally, using a bound analogous to the one given in (50) of the quantity $\max \left\{\left|\psi_{\lambda}(\mathrm{i} t)\right|,\left|z_{0}(t)\right|, 1\right\}$, we obtain the result for i $t \in D_{2} \cap E$ provided that $|\cos t| \leq$ $K_{1}|t-\mathrm{i} a|$ for some $K_{1}$. If i $t \in D_{3} \cap E$, we proceed in a similar way. We also can bound $\left|b_{2, j}\right|$ and $\left|b_{3, j}\right|$ and the lemma holds true.

The next lemma provides all the properties of $\mathcal{F}_{0}$ that we will use later on. We recall that $\mathcal{F}_{0}$ was defined in (64).

Lemma 3.8. If $p>-2$, the linear operator $I d-\mathcal{F}_{0}$ is invertible in $\mathcal{Z}$. Moreover, $\left\|\mathcal{F}_{0}\right\|_{\mathcal{Z}} \leq K \delta^{p+2}|\log \delta|$ and hence $\left\|\left(I d-\mathcal{F}_{0}\right)^{-1}\right\|_{\mathcal{Z}} \leq\left(1-\left\|\mathcal{F}_{0}\right\|_{\mathcal{Z}}\right)^{-1} \leq 1+K \delta^{p+2}|\log \delta|$.

Proof. During the proof of this lemma, we will denote by $K$ any constant independent of $\delta$.

Since $\mathcal{F}_{0}$ is a linear operator, to prove this lemma we only have to check that $\left\|\mathcal{F}_{0}\right\|_{\mathcal{Z}}<$ $K \delta^{p+2}|\log \delta|^{2}<1$, provided $p>-2$.

Let $h \in \mathcal{Z}$. We note that, by Lemma 3.7 and definition (74) of norm $\|\cdot\|_{\mathcal{Z}}$,

$$
\begin{aligned}
\left|\pi^{j}(B(\mathrm{i} t) h(\mathrm{i} t))\right| \leq & K \delta^{p+3}|\log \delta| \frac{1}{\cos ^{2} t} \mathrm{e}^{-\alpha(a-|t|) / \delta}\|h\|_{\mathcal{Z}} \\
& \times\left(2|\cos t|+|\log \delta| \delta^{p+4} \frac{1}{|\cos t|}\right)
\end{aligned}
$$


Breakdown of Heteroclinic Orbits

OF31

$$
\begin{aligned}
\leq & K \delta^{p+3}|\log \delta| \mathrm{e}^{-\alpha(a-|t|) / \delta}\|h\|_{\mathcal{Z}} \frac{1}{|\cos t|} \\
& \times\left(2+|\log \delta| \delta^{p+4} \frac{1}{\left|\cos ^{2} t\right|}\right) \\
\leq & K \delta^{p+3}|\log \delta| \mathrm{e}^{-\alpha(a-|t|) / \delta}\|h\|_{\mathcal{Z}} \frac{1}{|\cos t|}
\end{aligned}
$$

for $j=1,2,3$. In the last inequality we used that $p>-2$ and that $\delta$ is small enough. Therefore, we have that, for all i $t \in E$, that is, $|t| \leq a-\delta$,

$$
\begin{aligned}
\left|\pi^{1} \mathcal{G}(h)(\mathrm{i} t)\right| \leq & \mathrm{e}^{\alpha t / \delta} \mathrm{e}^{|c| \pi / 2} \cos t \int_{t}^{a-\delta} \frac{\mathrm{e}^{|c| \pi / 2}}{\cos r} \mathrm{e}^{-\alpha r / \delta}\left|\pi^{1}(B(\mathrm{i} r) h(\mathrm{i} r))\right| d r \\
\leq & K \delta^{p+3}|\log \delta| \mathrm{e}^{-\alpha a / \delta} \mathrm{e}^{\alpha t / \delta} \mathrm{e}^{|c| \pi} \cos t\|h\|_{\mathcal{Z}} \\
& \times \int_{t}^{a-\delta} \frac{1}{\cos ^{2} r} \mathrm{e}^{-\alpha(r-|r|) / \delta} d r .
\end{aligned}
$$

It is not difficult to check that there exists a constant $C$ independent of $\delta$ such that, for any $t \in[-(a-\delta),(a-\delta)]$,

$$
\mathrm{e}^{\alpha t / \delta} \int_{t}^{a-\delta} \frac{1}{\cos ^{2} r} \mathrm{e}^{-\alpha(r-|r|) / \delta} d r \leq C \mathrm{e}^{\alpha|t| / \delta} \delta^{-1} .
$$

Using the previous bound in (77), we have that

$$
\left\|\pi^{1} \mathcal{G}(h)\right\|_{\mathcal{Z}_{1}}=\sup _{\mathrm{i} t \in E}\left|\pi^{1} \mathcal{G}(h)(t) \mathrm{e}^{\alpha(a-|t|) / \delta} \cos ^{-1} t\right| \leq K \delta^{p+2}|\log \delta|\|h\|_{\mathcal{Z}}
$$

In the same way, one can check that

$$
\left\|\pi^{2} \mathcal{G}(h)\right\|_{\mathcal{Z}_{1}}=\sup _{\mathrm{i} t \in E}\left|\pi^{2} \mathcal{G}(h)(t) \mathrm{e}^{\alpha(a-|t|) / \delta} \cos ^{-1} t\right| \leq K \delta^{p+2}|\log \delta|\|h\|_{\mathcal{Z}}
$$

Now we deal with the operator $\mathcal{H}$. First we note that, by bounds (78) and (79), again using Lemma 3.7 to bound $b_{i, j}(t)$, we have that, since $|\cos t| \geq \delta$,

$$
\begin{aligned}
\left|\pi^{3}\left(B(\mathrm{i} t)\left(\mathcal{G}(h)(t), \pi^{3} h(\mathrm{i} t)\right)\right)\right| \leq & K \delta^{p+3}|\log \delta| \mathrm{e}^{-\alpha(a-|t|) / \delta} \frac{1}{\cos ^{2} t} \\
& \cdot\left(|\cos t|\left(\left\|\pi^{1} \mathcal{G}(h)\right\|_{\mathcal{Z}_{1}}+\left\|\pi^{2} \mathcal{G}(h)\right\|_{\mathcal{Z}_{1}}\right)\right. \\
& \left.+\frac{\delta^{p+4}|\log \delta|}{|\cos t|}\|h\|_{\mathcal{Z}_{2}}\right) \\
\leq & K \delta^{2 p+5}|\log \delta|^{2} \mathrm{e}^{-\alpha(a-|t|) / \delta}\|h\|_{\mathcal{Z}} \frac{1}{|\cos t|} .
\end{aligned}
$$

Then,

$$
|\mathcal{H}(h)(\mathrm{i} t)| \leq K \delta^{2 p+5}|\log \delta|^{2} \mathrm{e}^{-\alpha a / \delta}\|h\|_{\mathcal{Z}} \frac{1}{\cos ^{2} t}\left|\int_{0}^{t} \cos r \mathrm{e}^{\alpha|r| / \delta} d r\right|,
$$


and using that

$$
\begin{aligned}
\left|\int_{0}^{t} \cos r \mathrm{e}^{\alpha|r| / \delta} d r\right| & =\mathrm{e}^{\alpha|t| / \delta} \frac{\delta}{\alpha^{2}+\delta^{2}}(\alpha \cos t+\delta \sin |t|)-\alpha \frac{\delta}{\alpha^{2}+\delta^{2}} \\
& \leq C \frac{\delta}{\alpha} \mathrm{e}^{\alpha|t| / \delta} \cos t,
\end{aligned}
$$

for some constant $C>0$, we can prove that

$$
|\mathcal{H}(h)(\mathrm{i} t)| \leq K \delta^{2 p+6}|\log \delta|^{2} \mathrm{e}^{-\alpha(a-|t|) / \delta}\|h\|_{\mathcal{Z}} \frac{1}{|\cos t|} .
$$

Finally, using bounds (78), (79), and (82) and the definitions of $\mathcal{F}_{0}$ and $\|\cdot\|_{\mathcal{Z}}$, we have that

$$
\begin{aligned}
\left\|\mathcal{F}_{0}(h)\right\|_{\mathcal{Z}} & =\left\|\pi^{1} \mathcal{G}(h)\right\|_{\mathcal{Z}_{1}}+\left\|\pi^{2} \mathcal{G}(h)\right\|_{\mathcal{Z}_{1}}+\delta^{-p-4}|\log \delta|^{-1}\|\mathcal{H}(h)(t)\|_{\mathcal{Z}_{2}} \\
& \leq K \delta^{p+2}|\log \delta|\|h\|_{\mathcal{Z}} .
\end{aligned}
$$

Therefore, $\left\|\mathcal{F}_{0}\right\|_{\mathcal{Z}}<1$, provided that $p>-2$ and $\delta$ is small enough; this implies that the linear operator $\mathrm{Id}-\mathcal{F}_{0}$ is invertible and moreover $\left\|\left(\mathrm{Id}-\mathcal{F}_{0}\right)^{-1}\right\|_{\mathcal{Z}} \leq\left(1-\left\|\mathcal{F}_{0}\right\|_{\mathcal{Z}}\right)^{-1} \leq$ $1+K \delta^{p+2}|\log \delta|$.

3.3.2. End of the Proof of Proposition 3.3. At the beginning of Section 3.3, we explained our strategy to prove Proposition 3.3. The first step, the study of the linear operator $\mathcal{F}_{0}$, has been done in the previous subsection.

We recall that $\Delta \varphi_{1}=I\left(c_{1}^{0}, c_{2}^{0}\right)$, where $I$ was defined in (65). Taking into account this expression, we also notice that, by (69) in Lemma 3.6, we have that

$$
\left(\mathrm{Id}-\mathcal{F}_{0}\right) \Delta \varphi_{2}=I\left(c_{1}-c_{1}^{0}, c_{2}-c_{2}^{0}\right)+\mathcal{F}_{0}\left(I\left(c_{1}^{0}, c_{2}^{0}\right)\right) .
$$

It only remains to check that both $I\left(c_{1}-c_{1}^{0}, c_{2}, c_{2}^{0}\right)$ and $I\left(c_{1}^{0}, c_{2}^{0}\right)$ belong to $\mathcal{Z}$. This is done in the lemma below.

Lemma 3.9. Given $k_{1}, k_{2} \in \mathbb{C}, I\left(k_{1}, k_{2}\right) \in \mathcal{Z}$ and

$$
\left\|I\left(k_{1}, k_{2}\right)\right\|_{\mathcal{Z}} \leq K\left(\left|k_{1}\right|+\left|k_{2}\right|\right) \mathrm{e}^{\alpha a / \delta} .
$$

Proof. Throughout this proof, $K$ will denote any constant independent of $\delta$.

We fix $k_{1}, k_{2} \in \mathbb{C}$. It is clear that

$$
\left|k_{1}\right| \mathrm{e}^{\alpha a / \delta} \sup _{\mathrm{i} t \in E} \mathrm{e}^{\alpha t / \delta} \mathrm{e}^{-\alpha|t| / \delta}+\left|k_{2}\right| \mathrm{e}^{\alpha a / \delta} \sup _{\mathrm{i} t \in E} \mathrm{e}^{-\alpha t / \delta} \mathrm{e}^{-\alpha|t| / \delta}=\mathrm{e}^{\alpha a / \delta}\left(\left|k_{1}\right|+\left|k_{2}\right|\right) .
$$

In order to bound the third component of $I\left(k_{1}, k_{2}\right)$, we use bound (76) with $h=$ $\left(\pi^{1} I\left(k_{1}, k_{2}\right), \pi^{2} I\left(k_{1}, k_{2}\right), 0\right)$ and we obtain that, since $\|h\|_{\mathcal{Z}} \leq \mathrm{e}^{\alpha a / \delta} \mathrm{e}^{c \pi / 2}\left(\left|k_{1}\right|+\left|k_{2}\right|\right)$,

$$
\begin{aligned}
& \mid \pi^{3}(B(\mathrm{i} t)\left.\left(\pi^{1} I\left(k_{1}, k_{2}\right)(t), \pi^{2} I\left(k_{1}, k_{2}\right)(t), 0\right)\right) \mid \\
& \leq K \delta^{p+3}|\log \delta| \mathrm{e}^{\alpha|t| / \delta}\left(\left|k_{1}\right|+\left|k_{2}\right|\right) \frac{1}{|\cos t|} .
\end{aligned}
$$


Hence,

$$
\left|\pi^{3} I\left(k_{1}, k_{2}\right)(t)\right| \leq K \delta^{p+3}|\log \delta|\left(\left|k_{1}\right|+\left|k_{2}\right|\right) \frac{1}{\cos ^{2} t}\left|\int_{0}^{t} \cos r \mathrm{e}^{\alpha|r| / \delta} d r\right|,
$$

and finally, using (81) to bound the last integral, we get

$$
\left|\pi^{3} I\left(k_{1}, k_{2}\right)(t)\right| \leq K \delta^{p+4}|\log \delta|\left(\left|k_{1}\right|+\left|k_{2}\right|\right) \frac{1}{|\cos t|} \mathrm{e}^{\alpha|t| / \delta},
$$

and the result is proved since

$$
\begin{aligned}
\left\|I\left(k_{1}, k_{2}\right)\right\|_{\mathcal{Z}}= & \left\|\pi^{1} I\left(k_{1}, k_{2}\right)\right\|_{\mathcal{Z}_{1}}+\left\|\pi^{2} I\left(k_{1}, k_{2}\right)\right\|_{\mathcal{Z}_{1}} \\
& +|\log \delta|^{-1} \delta^{-p-4}\left\|\pi^{3} I\left(k_{1}, k_{2}\right)\right\|_{\mathcal{Z}_{2}} \\
\leq & K\left(\left|k_{1}\right|+\left|k_{2}\right|\right) \mathrm{e}^{\alpha a / \delta} .
\end{aligned}
$$

Lemma 3.10. $\Delta \varphi_{2} \in \mathcal{Z}$ and it is determined by

$$
\Delta \varphi_{2}=\left(I d-\mathcal{F}_{0}\right)^{-1}\left(I\left(c_{1}-c_{1}^{0}, c_{2}-c_{2}^{0}\right)\right)+\left(I d-\mathcal{F}_{0}\right)^{-1}\left(\mathcal{F}_{0}\left(\Delta \varphi_{1}\right)\right) .
$$

Moreover,

$$
\left\|\Delta \varphi_{2}\right\|_{\mathcal{Z}} \leq K \delta^{2 p+2}|\log \delta|
$$

Proof. We recall that $\Delta \varphi_{2}$ satisfies equation (69) given in Lemma 3.6; that is, we have that

$$
\left(\operatorname{Id}-\mathcal{F}_{0}\right)\left(\Delta \varphi_{2}\right)=I\left(c_{1}-c_{1}^{0}, c_{2}-c_{2}^{0}\right)+\mathcal{F}_{0}\left(\Delta \varphi_{1}\right) .
$$

Hence, since by Lemma 3.8 the operator Id $-\mathcal{F}_{0}$ is invertible in $\mathcal{Z}$, to prove (83) is equivalent to checking that both $I\left(c_{1}-c_{1}^{0}, c_{2}-c_{2}^{0}\right)$ and $\mathcal{F}_{0}\left(\Delta \varphi_{1}\right)$ belong to $\mathcal{Z}$. As in Lemma 3.9 we have proved that $I\left(k_{1}, k_{2}\right) \in \mathcal{Z}$ for all $k_{1}, k_{2} \in \mathbb{C}$, the functions $I\left(c_{1}-c_{1}^{0}, c_{2}-c_{2}^{0}\right)$ and $\Delta \varphi_{1}=I\left(c_{1}^{0}, c_{2}^{0}\right)$ belong to $\mathcal{Z}$. Moreover, in Lemma 3.8, we saw that $\mathcal{F}_{0}(\mathcal{Z}) \subset \mathcal{Z}$, and hence $\mathcal{F}_{0}\left(\Delta \varphi_{1}\right) \in \mathcal{Z}$, which implies that $\Delta \varphi_{2} \in \mathcal{Z}$.

Now we will prove the second part of the lemma: the bound of $\left\|\Delta \varphi_{2}\right\|_{\mathcal{Z}}$. Since $\Delta \varphi_{2}$ satisfies the identity (83),

$$
\left\|\Delta \varphi_{2}\right\|_{\mathcal{Z}} \leq\left\|\left(\operatorname{Id}-\mathcal{F}_{0}\right)^{-1}\right\|_{\mathcal{Z}}\left(\left\|I\left(c_{1}-c_{1}^{0}, c_{2}-c_{2}^{0}\right)\right\|_{\mathcal{Z}}+\left\|\mathcal{F}_{0}\right\|_{\mathcal{Z}}\left\|\Delta \varphi_{1}\right\|_{\mathcal{Z}}\right) .
$$

Taking into account bound (63) of $c_{1}^{0}, c_{2}^{0}$, and bound (70) of Lemma 3.6 to estimate $c_{1}-c_{1}^{0}, c_{2}-c_{2}^{0}$, we are able to bound $\Delta \varphi_{1}=I\left(c_{1}^{0}, c_{2}^{0}\right)$ and $I\left(c_{1}-c_{1}^{0}, c_{2}-c_{2}^{0}\right)$ by using Lemma 3.9. We also bound $\left\|\mathcal{F}_{0}\right\|_{\mathcal{Z}}$ and $\left\|\left(\operatorname{Id}-\mathcal{F}_{0}\right)^{-1}\right\|_{\mathcal{Z}}$ by using Lemma 3.8 and finally we obtain that, if $\delta$ is small enough,

$$
\begin{aligned}
\left\|\Delta \varphi_{2}\right\|_{\mathcal{Z}} & \leq 2 K \mathrm{e}^{\alpha a / \delta}\left(\left|c_{1}-c_{1}^{0}\right|+\left|c_{2}-c_{2}^{0}\right|\right)+K \delta^{p+2}|\log \delta| \mathrm{e}^{\alpha a / \delta}\left(\left|c_{1}^{0}\right|+\left|c_{2}^{0}\right|\right) \\
& \leq K \delta^{2 p+2}|\log \delta|,
\end{aligned}
$$

and the lemma is proved. 
End of the proof of Proposition 3.3. We note that, since $\pi^{3} \Delta \varphi(0)=\pi^{3} \Delta \varphi_{1}(0)=0$, then $\pi^{3} \Delta \varphi_{2}(0)=0$. Moreover, by Lemma 3.10 and definition (74) of $\|\cdot\|_{\mathcal{Z}}$, it is clear that

$$
\left|\pi^{1,2} \Delta \varphi_{2}(0)\right| \leq K \delta^{2 p+2}|\log \delta| \mathrm{e}^{-\alpha a / \delta},
$$

and Proposition 3.3 is proved.

\subsection{Proof of Proposition 3.4}

We recall that $\pi^{3} \varphi_{1}(0)=0$, and hence we only have to compute $\Delta \psi_{1}(0)=\pi^{1,2} \Delta \varphi_{1}(0)$. We also notice that

$$
\Delta \psi_{1}(0)=\left(c_{1}^{0}, c_{2}^{0}\right)^{\mathrm{T}}
$$

where $c_{1}^{0}$ and $c_{2}^{0}$ were defined in (61). Moreover, by Lemma 3.1, $c_{1}^{0}=\overline{c_{2}^{0}}$; hence, we only have to calculate $c_{1}^{0}$.

We recall that by definition (11) of $\mathcal{M}$, we have $\pi^{1} \mathcal{M}(0)=\delta^{p} F_{1}\left(0,0, \delta z_{0}(t), \delta\right)=$ $\delta^{p}(f+\mathrm{i} g)\left(0,0, \delta z_{0}(t), \delta\right)$. Moreover, since $(f, g)(0,0, \delta z, \delta)=O\left(|(\delta z, \delta)|^{3}\right)$, one has

$$
(f+\mathrm{i} g)(0,0, \delta z, \delta)=\delta^{p+3} a_{0}(\delta)+\delta^{p+3} a_{1}(\delta) z+\delta^{p+3} a_{2}(\delta) z^{2}+\sum_{n \geq 3} \delta^{p+n} a_{n}(\delta) z^{n},
$$

where $a_{n}$ are bounded and analytic functions in $B\left(\delta_{0}\right)$. Consequently,

$$
\begin{aligned}
\pi^{1} \mathcal{M}(0)(t)= & \delta^{p+3} a_{0}(\delta)-\delta^{p+3} a_{1}(\delta) \tanh t+\delta^{p+3} a_{2}(\delta) \tanh ^{2} t \\
& +\sum_{n \geq 3} \delta^{p+n}(-1)^{n} a_{n}(\delta) \tanh ^{n} t .
\end{aligned}
$$

We denote

$$
I_{n}:=I_{n}(\alpha, c)=\int_{-\infty}^{+\infty} \frac{\sinh ^{n} r}{(\cosh r)^{n+1+\mathrm{i} c}} \mathrm{e}^{\mathrm{i} \alpha r / \delta} d r
$$

and we observe that, by expression (86) of $\mathcal{M}(0)$ and definition (61) of $c_{1}^{0}$, we have that

$$
c_{1}^{0}=\delta^{p+3} a_{0}(\delta) I_{0}-\delta^{p+3} a_{1}(\delta) I_{1}+\delta^{p+3} a_{2}(\delta) I_{2}+\sum_{n \geq 3} \delta^{p+n}(-1)^{n} a_{n}(\delta) I_{n} .
$$

To get the asymptotic expression of Proposition 3.4, we have to estimate $I_{n}$. First we claim that $I_{n}$ satisfies the recurrence relation

$$
I_{n}=\frac{1}{n+\mathrm{i} c} \frac{\mathrm{i} \alpha}{\delta} I_{n-1}+\frac{n-1}{n+\mathrm{i} c} I_{n-2} .
$$

The claim follows easily by doing parts in definition (87) of $I_{n}$. We define the sequence $\left\{J_{n}\right\}_{n \geq 0}$ by

$$
J_{n}=\frac{1}{n+\mathrm{i} c} \frac{\mathrm{i} \alpha}{\delta} J_{n-1}, \quad J_{0}=I_{0}
$$


and we claim that $I_{n}=J_{n}\left(1+\bar{I}_{n}\right)$ with $\bar{I}_{n}$ satisfying that $\bar{I}_{0}=\bar{I}_{1}=0$ and $\left|\bar{I}_{n}\right| \leq$ $\delta|\Gamma(n+1+\mathrm{i} c)|$. Indeed, it is clear that $I_{1}=J_{1}$ and that $\bar{I}_{n}$ satisfies the recurrence relation given by

$$
\bar{I}_{n}=\bar{I}_{n-1}+(n-1)(n-1+\mathrm{i} c)\left(\frac{\delta}{\alpha \mathrm{i}}\right)^{2}\left(1+\bar{I}_{n-2}\right), \quad \bar{I}_{0}=\bar{I}_{1}=0 .
$$

Now we proceed by induction. Let us assume that $\left|\bar{I}_{k}\right| \leq \delta|\Gamma(k+1+\mathrm{i} c)|$ for all $k<n$. Henceforth,

$$
\begin{aligned}
\bar{I}_{n} & \leq \delta|\Gamma(n+\mathrm{i} c)|+(n-1)|n-1+\mathrm{i} c| \frac{\delta^{2}}{\alpha^{2}}(1+\delta|\Gamma(n-1+\mathrm{i} c)|) \\
& =\delta|\Gamma(n+\mathrm{i} c)|\left(1+\frac{\delta^{2}}{\alpha^{2}}(n-1)\right)+(n-1)|n-1+\mathrm{i} c| \frac{\delta^{2}}{\alpha^{2}} \\
& =\delta|\Gamma(n+\mathrm{i} c)|\left(1+\frac{\delta^{2}}{\alpha^{2}}(n-1)+\frac{n-1}{|\Gamma(n-1+\mathrm{i} c)|} \frac{\delta}{\alpha^{2}}\right) \\
& \leq \delta|\Gamma(n+\mathrm{i} c)| n \leq \delta|\Gamma(n+1+\mathrm{i} c)|,
\end{aligned}
$$

if $\delta$ is small enough, but independent of $n$. Here we have used that $\Gamma(z+1)=z \Gamma(z)$ and that $n /|\Gamma(n+\mathrm{i} c)|$ is bounded for all $n$.

Now we are going to estimate $J_{n}$. We have that

$$
J_{n}=\prod_{k=1}^{n} \frac{1}{k+\mathrm{i} c}\left(\frac{\alpha \mathrm{i}}{\delta}\right)^{n} J_{0}=\left(\frac{\alpha \mathrm{i}}{\delta}\right)^{n} \frac{\Gamma(1+\mathrm{i} c)}{\Gamma(n+1+\mathrm{i} c)} J_{0}, \quad n \geq 1 .
$$

Performing the change of variables $s=\tanh r$, we get that

$$
J_{0}=\int_{-\infty}^{+\infty} \frac{1}{(\cosh r)^{1+\mathrm{i} c}} \mathrm{e}^{\alpha \mathrm{i} r / \delta} d r=\int_{-1}^{1}(1+s)^{(-1+\mathrm{i} c) / 2+\alpha \mathrm{i} /(2 \delta)}(1-s)^{(-1+\mathrm{i} c) / 2-\alpha \mathrm{i} /(2 \delta)} d s .
$$

This integral can be expressed as a confluent hypergeometric function (see page 505, [AS92] for the definition):

$$
\begin{aligned}
M(a, b, z)= & \frac{\Gamma(b)}{\Gamma(b-a) \Gamma(a)} 2^{1-b} \mathrm{e}^{z / 2} \\
& \times \int_{-1}^{1} \mathrm{e}^{-z t / 2}(1+t)^{b-a-1}(1-t)^{a-1} d t, \quad \operatorname{Re} b>\operatorname{Re} a>0,
\end{aligned}
$$

taking $a=(1+\mathrm{i} c) / 2-\mathrm{i} \alpha /(2 \delta), b=1+\mathrm{i} c$, and $z=0$. It is well known that $M(a, b, 0)=1$. Hence, we have that

$$
J_{0}=2^{\mathrm{i} c} \frac{1}{\Gamma(1+\mathrm{i} c)} \Gamma\left(\frac{1+\mathrm{i} c}{2}+\frac{\alpha \mathrm{i}}{2 \delta}\right) \Gamma\left(\frac{1+\mathrm{i} c}{2}-\frac{\alpha \mathrm{i}}{2 \delta}\right),
$$

and thus, substituting the previous expression of $J_{0}$ in (89), we have that

$$
J_{n}=\left(\frac{\mathrm{i} \alpha}{\delta}\right)^{n} \frac{1}{\Gamma(n+1+\mathrm{i} c)} 2^{\mathrm{i} c} \Gamma\left(\frac{1+\mathrm{i} c}{2}+\frac{\alpha \mathrm{i}}{2 \delta}\right) \Gamma\left(\frac{1+\mathrm{i} c}{2}-\frac{\alpha \mathrm{i}}{2 \delta}\right) .
$$


Now we use that for any $z \in \mathbb{C}$ such that $|\arg (z)|<\pi, \Gamma(z+a)=\Gamma(z+b) z^{a-b}(1+$ $O\left(z^{-1}\right)$ ) for arbitrary $a, b \in \mathbb{C}$ (see [EMOT53], page 47), we obtain that

$$
\Gamma\left(\frac{1+\mathrm{i} c}{2} \pm \frac{\alpha \mathrm{i}}{2 \delta}\right)=\Gamma\left(\frac{1}{2} \pm \frac{\alpha \mathrm{i}}{2 \delta}\right)\left( \pm \frac{\alpha \mathrm{i}}{2 \delta}\right)^{\mathrm{i} c / 2}(1+O(\delta))
$$

(taking $z= \pm \alpha \mathrm{i} /(2 \delta), a=(1+\mathrm{i} c) / 2$ and $b=1 / 2)$, and thus, using that $\Gamma(z) \Gamma(\bar{z})=$ $|\Gamma(z)|^{2}$, from (90) we have that

$$
\begin{aligned}
J_{n} & =\left(\frac{\mathrm{i} \alpha}{\delta}\right)^{n} \frac{1}{\Gamma(n+1+\mathrm{i} c)}\left|\Gamma\left(\frac{1}{2}+\frac{\mathrm{i}|\alpha|}{2 \delta}\right)\right|^{2} 2^{\mathrm{i} c}\left|\frac{\mathrm{i}|\alpha|}{2 \delta}\right|^{\mathrm{i} c}(1+O(\delta)) \\
& =\mathrm{i}^{n}\left(\frac{\alpha}{\delta}\right)^{n}\left(\frac{|\alpha|}{\delta}\right)^{\mathrm{i} c} \frac{1}{\Gamma(n+1+\mathrm{i} c)}\left|\Gamma\left(\frac{1}{2}+\frac{\mathrm{i}|\alpha|}{2 \delta}\right)\right|^{2}(1+O(\delta)),
\end{aligned}
$$

where $O(\delta)$ is independent of $n$. Finally, using that $\Gamma(z)=\mathrm{e}^{-z} \mathrm{e}^{(z-1 / 2) \log z}(2 \pi)^{1 / 2}(1+$ $O\left(z^{-1}\right)$ ) for any $z \in \mathbb{C}$ with $|\arg z|<\pi$, we have that, if $\delta$ is small enough,

$$
\left|\Gamma\left(\frac{1}{2}+\frac{\mathrm{i}|\alpha|}{2 \delta}\right)\right|^{2}=2 \pi \mathrm{e}^{-\pi|\alpha| /(2 \delta)}(1+O(\delta)) .
$$

Therefore, using (92) in (91), we obtain that for any $\alpha, c J_{n}$ can be expressed as

$$
J_{n}=\mathrm{i}^{n}\left(\frac{\alpha}{\delta}\right)^{n}\left(\frac{|\alpha|}{\delta}\right)^{\mathrm{i} c} \frac{2 \pi}{\Gamma(n+1+\mathrm{i} c)} \mathrm{e}^{-\pi|\alpha| /(2 \delta)}(1+O(\delta)),
$$

and therefore

$$
\begin{aligned}
I_{n}(\alpha, c) & =\mathrm{i}^{n}\left(\frac{\alpha}{\delta}\right)^{n}\left(\frac{|\alpha|}{\delta}\right)^{\mathrm{i} c} \frac{2 \pi}{\Gamma(n+1+\mathrm{i} c)} \mathrm{e}^{-\pi|\alpha| /(2 \delta)}(1+O(\delta))\left(1+\bar{I}_{n}(\alpha, c)\right) \\
& =\mathrm{i}^{n}\left(\frac{\alpha}{\delta}\right)^{n}\left(\frac{|\alpha|}{\delta}\right)^{\mathrm{i} c} \frac{2 \pi}{\Gamma(n+1+\mathrm{i} c)} \mathrm{e}^{-\pi|\alpha| /(2 \delta)}+O\left(\delta^{1-n}\right) \mathrm{e}^{-\pi|\alpha| /(2 \delta)},
\end{aligned}
$$

provided that $\bar{I}_{n}(\alpha, c)$ satisfy that $\left|\bar{I}_{n}(\alpha, c)\right| \leq \delta|\Gamma(n+1+\mathrm{i} c)|$.

To finish the proof of Proposition 3.4, we introduce $m_{n}=(-1)^{n} a_{n}(0)$, for $n \geq 3$. If we substitute the above asymptotic expression in equality (88) and we take into account that $I_{n}=O\left(\delta^{-n} \mathrm{e}^{-\pi|\alpha| /(2 \delta)}\right)$, we obtain an asymptotic formula for $c_{1}^{0}$. Then, we only need to use the fact that, by $(84), \pi^{1,2} \varphi_{1}(0)=\Delta \psi_{1}(0)=\left(c_{1}^{0}, c_{2}^{0}\right)^{\mathrm{T}}$.

\section{Conclusions}

The main result in this paper is Theorem 1.4, where an asymptotic formula for the exponentially small distance between the (one-dimensional) unstable and stable manifolds of the two critical points of family (5) is obtained rigorously.

This result is a first step towards the complete understanding of the Hopf-zero singularity of vector fields in $\mathbb{R}^{3}$. The application, of the result obtained in this paper, to 
families $X_{\mu}$ of conservative vector fields in $\mathbb{R}^{3}$ that meet the singularity for some value of the parameter $\mu=\mu_{0}$, is of use when considering the behavior for $\mu$ close to $\mu_{0}$. In particular, one expects these families to experience a Shilnikov bifurcation, that is, the existence of a homoclinic orbit to one of the critical points, and chaos, for values of the parameter arbitrarily close to $\mu_{0}$.

Of course, the result in this paper is only a small contribution to the whole problem of the Hopf-zero singularity, and there are some challenging problems that can be naturally considered and solved by using these, and more sophisticated, techniques.

In the case of the heteroclinic connection considered in this paper, it is clear that the next goal is to prove conditions for its break-up in the case $p=-2$. The derivation of an asymptotic formula in this case will solve the problem for generic unfoldings. As we explained in Section 1.4, to deal with this case, we have to obtain suitable approximations for the invariant manifolds in complex domains which no longer form the unperturbed heteroclinic connection. These approximations are special solutions of the so-called inner system and one expects the distance between the perturbed invariant manifolds be dominated by their difference. In their study of the "inner system," [BS] proved the existence of suitable approximations of the invariant manifolds as well as give an asymptotic formula for their difference that depends on the full jet of $f+\mathrm{i} g$ in (1).

Of course, the study of the inner system does not solve the singular problem at all. In this case, the most difficult part will be the derivation of an asymptotic formula for the break-up, naturally different from the one obtained in this paper, and that involves, as the leading term, the results for the inner system. The authors plan to estimate the formula and to check it numerically before trying to apply matching techniques to prove it.

On the other hand, to obtain the existence of homoclinic orbits for these families (and therefore Shilnikov bifurcations), for both $p>-2$ and $p=-2$, it is not enough to prove the break-up of the heteroclinic orbit between the critical points. The reason is that, as was stated in Lemma 1.2, the close integrable system we use as an unperturbed system has a heteroclinic surface that is the two-dimensional stable and unstable manifolds associated with these critical points. Then, it is necessary to show that, when the higher order terms of the family are considered, these coincident two-dimensional manifolds also break up and intersect transversally along one (or two) heteroclinic orbits. We expect similar results to the one in this paper, validating the prediction of the Melnikov vector when $p>-2$ and a more difficult task also in the singular case $p=-2$.

There is a long way yet to go to achieve the complete result, and the work presented in this paper is only a first step. However, the result in this paper is also interesting in its own right, mainly because it deals with an unusual case, autonomous vector fields in $\mathbb{R}^{3}$ where these exponentially small phenomena occur. Finally, we point out that the techniques used in this paper are more in the spirit of the first work in this area [HMS88] because they do not use any geometric properties of the system considered.

\section{Acknowledgments}

This work has been supported by the Catalan grant 2001SGR-70 and the MCyT-FEDER grant BFM2003-9504. 


\section{References}

$\left[\mathrm{AMF}^{+} 03\right]$ A. Algaba, M. Merino, E. Freire, E. Gamero, and A. J. Rodríguez-Luis. Some results on Chua's equation near a triple-zero linear degeneracy. Int. J. Bifur. Chaos Appl. Sci. Eng., 13(3):583-608, 2003.

[Ang93] S. Angenent. A variational interpretation of Mel'nikov's function and exponentially small separatrix splitting. In Symplectic Geometry, volume 192 of London Math. Soc. Lecture Note Ser., pages 5-35. Cambridge Univ. Press, Cambridge, 1993.

[AS92] M. Abramowitz and I. A. Stegun, editors. Handbook of Mathematical Functions with Formulas, Graphs, and Mathematical Tables. Dover Publications Inc., New York, 1992. Reprint of the 1972 edition.

[Bal] I. Baldomá. The inner equation for one and a half degrees of freedom rapidly forced Hamiltonian systems. Nonlinearity, 19:1415-1445, 2006.

[BF04] I. Baldomá and E. Fontich. Exponentially small splitting of invariant manifolds of parabolic points. Mem. Amer. Math. Soc., 167(792):x-83, 2004.

[BS] I. Baldomá and T.M. Seara. The inner equation for analytic unfoldings of the hopfzero singularity. preprint.

[Bro81a] H. Broer. Formal normal form theorems for vector fields and some consequences for bifurcations in the volume preserving case. In Dynamical Systems and Turbulence, Warwick 1980 (Coventry, 1979/1980), volume 898 of Lecture Notes in Math., pages 54-74. Springer, Berlin, 1981.

[Bro81b] H. Broer. Quasiperiodic flow near a codimension one singularity of a divergence free vector field in dimension three. In Dynamical Systems and Turbulence, Warwick 1980 (Coventry, 1979/1980), volume 898 of Lecture Notes in Math., pages 75-89. Springer, Berlin, 1981.

[BV84] H. W. Broer and G. Vegter. Subordinate Šil'nikov bifurcations near some singularities of vector fields having low codimension. Ergodic Theory Dynam. Syst., 4(4):509$525,1984$.

[DG00] A. Delshams and P. Gutiérrez. Splitting potential and the Poincaré-Melnikov method for whiskered tori in Hamiltonian systems. J. Nonlin. Sci., 10(4):433-476, 2000.

[DGJS97] A. Delshams, V. Gelfreich, A. Jorba, and T. M. Seara. Exponentially small splitting of separatrices under fast quasiperiodic forcing. Commun. Math. Phys., 189(1):35-71, 1997.

[DI98] F. Dumortier and S. Ibáñez. Singularities of vector fields on $\mathbf{R}^{3}$. Nonlinearity, 11(4):1037-1047, 1998.

[DRR98] A. Delshams and R. Ramírez-Ros. Exponentially small splitting of separatrices for perturbed integrable standard-like maps. J. Nonlin. Sci., 8(3):317-352, 1998.

[DS97] A. Delshams and T. M. Seara. Splitting of separatrices in Hamiltonian systems with one and a half degrees of freedom. Math. Phys. Electron. J., 3:Paper 4, 40 pp. (electronic), 1997.

[Eli94] L. H. Eliasson. Biasymptotic solutions of perturbed integrable Hamiltonian systems. Bol. Soc. Brasil. Mat. (N.S.), 25(1):57-76, 1994.

[EMOT53] A. Erdélyi, W. Magnus, F. Oberhettinger, and F. G. Tricomi. Higher Transcendental Functions. Vol. I. McGraw-Hill Book Company, Inc., New York-Toronto-London, 1953. Based, in part, on notes left by Harry Bateman.

[FGRLA02] E. Freire, E. Gamero, A. J. Rodríguez-Luis, and A. Algaba. A note on the triplezero linear degeneracy: Normal forms, dynamical and bifurcation behaviors of an unfolding. Int. J. Bifur. Chaos Appl. Sci. Eng., 12(12):2799-2820, 2002.

[Ge197a] V. G. Gelfreich. Melnikov method and exponentially small splitting of separatrices. Physica D, 101(3-4):227-248, 1997.

[Ge197b] V. G. Gelfreich. Reference systems for splittings of separatrices. Nonlinearity, 10(1):175-193, 1997. 
[GH83] J. Guckenheimer and P. Holmes. Nonlinear Oscillations, Dynamical Systems, and Bifurcations of Vector Fields, volume 42 of Applied Mathematical Sciences. SpringerVerlag, New York, 1983.

[GS01] V. Gelfreich and D. Sauzin. Borel summation and splitting of separatrices for the Hénon map. Ann. Inst. Fourier (Grenoble), 51(2):513-567, 2001.

[Guc81] J. Guckenheimer. On a codimension two bifurcation. In Dynamical Systems and Turbulence, Warwick 1980 (Coventry, 1979/1980), volume 898 of Lecture Notes in Math., pages 99-142. Springer, Berlin, 1981.

[HMS88] P. Holmes, J. Marsden, and J. Scheurle. Exponentially small splittings of separatrices with applications to KAM theory and degenerate bifurcations. In Hamiltonian Dynamical Systems (Boulder, CO, 1987), volume 81 of Contemp. Math., pages 213244. Amer. Math. Soc., Providence, RI, 1988.

[JTM92] J. Jones, W. C. Troy, and A. D. MacGillivray. Steady solutions of the KuramotoSivashinsky equation for small wave speed. J. Diff. Eq., 96(1):28-55, 1992.

[KT76] Y. Kuramoto and T. Tsuzuki. Persistent propagation of concentration waves in dissipative media far from thermal equilibrium. Prog. Theor. Phys., 55:356-369, 1976.

[Laz03] V. F. Lazutkin. Splitting of separatrices for the Chirikov standard map. Zap. Nauchn. Sem. S.-Peterburg. Otdel. Mat. Inst. Steklov. (POMI), 300 (Teor. Predst. Din. Sist. Spets. Vyp. 8):25-55, 285, 2003. Translated from the Russian and with a preface by V. Gelfreich, Workshop on Differential Equations (Saint Petersburg, 2002).

[Lom99] E. Lombardi. Nonpersistence of homoclinic connections for perturbed integrable reversible systems. J. Dynam. Diff. Eq., 11(1):129-208, 1999.

[LTW04] J. W. Lamb, M.A. Teixeira, and K. Webster. Heteroclinic bifurcations near Hopf-zero bifurcation in reversible vector fields in $\mathbf{R}^{3}$. J. Diff. Eq., 219(1):78-115.

[Mel63] V.F. Melnikov. On the stability of the center for time periodic perturbations. Trans. Moscow Math. Soc., 12:3-56, 1963.

[Mic86] D. Michelson. Steady solutions of the Kuramoto-Sivashinsky equation. Physica D, 19(1):89-111, 1986.

[OSS03] C. Olivé, D. Sauzin, and T. M. Seara. Resurgence in a Hamilton-Jacobi equation. In Proceedings of the International Conference in Honor of Frédéric Pham (Nice, 2002), volume 53, pages 1185-1235, 2003.

[Poi90] H. Poincaré. Sur le problème des trois corps et les équations de la dynamique. Acta Math., 13:1-271, 1890.

[RMT97] S. V. Raghavan, J. B. McLeod, and W. C. Troy. A singular perturbation problem arising from the Kuramoto-Sivashinsky equation. Diff. Int. Eq., 10(1):1-36, 1997.

[Sau01] D. Sauzin. A new method for measuring the splitting of invariant manifolds. Ann. Sci. École Norm. Sup. (4), 34(2):159-221, 2001.

[Sch89] J. Scheurle. Chaos in a rapidly forced pendulum equation. In Dynamics and Control of Multibody Systems (Brunswick, ME, 1988), volume 97 of Contemp. Math., pages 411-419. Amer. Math. Soc., Providence, RI, 1989.

[Šil65] L. P. Šil'nikov. A case of the existence of a countable number of periodic motions. Soviet Math. Dokl, 6:163-166, 1965.

[Šil70] L. P. Śil'nikov. On the question of the structure of an extended neighborhood of a structurally stable state of equilibrium of saddle-focus type. Mat. Sb. (N.S.), 81 (123):92-103, 1970.

[SSTC01] L. P. Shilnikov, A. L. Shilnikov, D. Turaev, and L. O. Chua. Methods of Qualitative Theory in Nonlinear Dynamics. Part II, volume 5 of World Scientific Series on Nonlinear Science. Series A: Monographs and Treatises. World Scientific Publishing Co. Inc., River Edge, NJ, 2001.

[Tak73a] F. Takens. A nonstabilizable jet of a singularity of a vector field. In Dynamical Systems (Proc. Sympos., Univ. Bahia, Salvador, 1971), pages 583-597. Academic Press, New York, 1973. 
[Tak73b] F. Takens. Normal forms for certain singularities of vector fields. Ann. Inst. Fourier (Grenoble), 23(2):163-195, 1973. Colloque International sur l'Analyse et la Topologie Différentielle (Colloques Internationaux du Centre National de la Recherche Scientifique, Strasbourg, 1972).

[Tak74] F. Takens. Singularities of vector fields. Inst. Hautes Études Sci. Publ. Math., (43):47100,1974

[Tre97] D. V. Treschev. Splitting of separatrices for a pendulum with rapidly oscillating suspension point. Russian J. Math. Phys., 5(1):63-98 (1998), 1997. 